\title{
Statistical field theory and effective action method for scalar active matter
}

\author{
M. Paoluzzi ${ }^{1,2, *}$ C. Maggi, ${ }^{3,2}$ and A. Crisanti ${ }^{2,1}$ \\ ${ }^{1}$ ISC-Consiglio Nazionale delle Ricerche, Institute for Complex Systems, Piazzale A. Moro 2, I-00185 Rome, Italy \\ ${ }^{2}$ Dipartimento di Fisica, Sapienza University of Rome, Piazzale A. Moro 2, I-00185 Rome, Italy \\ ${ }^{3}$ Institute of Nanotechnology (Consiglio Nazionale delle Ricerche-NANOTEC), Soft and Living Matter Laboratory, \\ Piazzale A. Moro 2, I-00185 Rome, Italy
}

(Received 24 September 2019; accepted 14 April 2020; published 21 May 2020)

\begin{abstract}
We employ statistical field theory techniques for coarse graining the steady-state properties of active OrnsteinUhlenbeck particles. The computation is carried out in the framework of the unified colored noise approximation that allows an effective equilibrium picture. We thus develop a mean-field theory that allows us to describe in a unified framework the phenomenology of scalar active matter. In particular, we are able to describe through a spontaneous symmetry-breaking mechanism two peculiar features of active systems: (i) the accumulation of active particles at the boundaries of a confining container and (ii) motility-induced phase separation (MIPS). We develop a mean-field theory for steric interacting active particles undergoing MIPS and for active Lennard-Jones (ALJ) fluids. Within this framework, we discuss the universality class of MIPS and ALJ fluids, showing that it falls into the Ising universality class. We thus compute analytically the critical line $T_{c}(\tau)$ for both models. In the case of MIPS, $T_{c}(\tau)$ gives rise to a reentrant phase diagram compatible with an inverse transition from liquid to gas as the strength of the noise decreases. However, in the case of particles interacting through anisotropic potentials, the field theory acquires a $\varphi^{3}$ term that, in general, cannot be canceled performing the expansion around the critical point. In this case, the Ising critical point might be replaced by a first-order phase-transition region.
\end{abstract}

DOI: 10.1103/PhysRevResearch.2.023207

\section{INTRODUCTION}

In nature, there are many diverse examples of living materials [1] ranging from epithelial monolayers [2] to bacterial colonies [3] to dense drops of ants [4]. Even though the elementary units composing such materials are complex biological objects, many of the emerging collective behaviors can be described using concepts of condensed matter and, in particular, through the analytical and numerical tools developed during the last decades in active matter [5-9]. Active systems are defined as a class of nonequilibrium systems consisting of interacting entities that individually dissipate energy to generate forces and motion and exhibit self-organized behavior at large scales.

Active systems can develop complex patterns that change dynamically, as in the case of flocking [9]. Flocking has been observed at very different scales ranging from birds [10] to epithelial monolayers [11]. However, active systems can also give rise to pattern formation and condensed phases with structural properties remarkably similar to those of ordinary materials, e.g., gas-liquid phase transition [12-15], glassy or jamming states [16-19], polar order [20], and nematic order [21]. Pattern formation in active matter is driven by out-of-equilibrium dynamics and thus these condensed phases

\footnotetext{
*matteo.paoluzzi@roma1.infn.it
}

Published by the American Physical Society under the terms of the Creative Commons Attribution 4.0 International license. Further distribution of this work must maintain attribution to the author(s) and the published article's title, journal citation, and DOI. are emergent properties of steady-state configurations that, in general, cannot be described by a Boltzmann distribution. In particular, the dependency on the microscopic dynamics makes it hard to establish possible universality classes. Understanding how condensed phases in active systems are related to those in equilibrium plays an important role in both basic and applied science. In basic science, it would allow us to gain insight into the concept of universality in nonequilibrium systems. In applied science, for instance, it would allow us to design living and synthetic materials with desired structural properties.

In this paper, we present a study on universal properties of a specific class of active matter systems that is described on a large scale by a scalar field theory. We start from a microscopic model where particles are self-propelled through a persistent noise [22-24] and we perform a coarse graining using the machinery of statistical field theory. We show that, within the framework of the unified colored noise (UCN) approximation [25,26], the structural properties of the system can be described through an opportune effective action [27]. Active field theories based on the dynamical evolution of opportune sets of order parameters have been largely employed for capturing the large-scale behavior of active systems [28-34]. In our paper, we show that some peculiar behaviors of active systems can be captured through an equilibrium statistical field theory approach.

\section{A. Summary of results}

We aim to develop a field theoretical description of active systems using the machinery of equilibrium statistical physics. We restrict our study to the case of a scalar theory, 
meaning that we implicitly assume that the relevant observable describing the system on a large scale is the density field. This assumption is reasonable if one is interested in a meanfield description of the structural properties at intermediate densities. It is worth noting that, for addressing crystallization in two dimensions, we should include orientational order parameters for discussing the role played by topological defects [35-37].

As a main result, we obtain that a scalar active system can be described by an effective action that counts nonlocal terms. The effective action is a functional of the scalar order parameter $\varphi$. These nonlocal terms can be systematically studied at the mean-field level.

Focusing our attention on mean-field computations, we show the following.

(i) The effective action method reproduces some basic properties of scalar active matter.

(ii) We can put into a unified theoretical framework two peculiar phenomena of active matter: the accumulation of active particles at the boundaries of a container and motilityinduced phase separation (MIPS) [38]. In particular, both phenomena can be interpreted in terms of a spontaneous symmetry breaking of $\varphi \rightarrow-\varphi$ symmetry.

(iii) We can discuss in a simple picture the universality class of MIPS and active Lennard-Jones (ALJ) fluids. In particular, in both cases, we can compute analytically the critical line $T_{c}(\tau)$. Moreover, mean-field theory suggests that both MIPS and ALJ fluids fall into the Ising universality class.

(iv) In the case of MIPS, the curve $T_{c}(\tau)$ develops a reentrant phase diagram indicating that the system undergoes an inverse transition from liquid to gas as the effective temperature $T$ is decreased above a threshold value $\tau_{\text {th }}$. This means that the condensed MIPS phase evaporates as $T$ decreases.

(v) We show that, close to the critical point, the effective action explicitly breaks the symmetry $\varphi \rightarrow-\varphi$ through a $\varphi^{3}$ term that vanishes for spherical active particles and is nonzero for anisotropic pair potentials, i.e., rod-shaped swimmers. Moreover, although this term does not touch the Ising universality class of the critical point, the presence of this term suggests that the MIPS critical point can be hidden by a first-order phase transition.

The paper is organized as follows. In Sec. II we introduce the theoretical framework. In Sec. III we define the microscopical model. In Sec. IV we employ the theoretical setup for studying one-body interactions in active matter. In particular, we show how the accumulation of active particles at the boundaries of a container can be interpreted as a spontaneous symmetry breaking in the effective action. In Sec. V we address many-body interactions and discuss the general features of the theory. In Sec. VI we discuss the mean-field approximation. In particular, we study the mean-field phase diagram of purely repulsive potentials and Lennard-Jones potentials. In Sec. VII we discuss the effect of anisotropic interaction on MIPS. Finally, in Sec. VIII we present our conclusions.

\section{THEORETICAL SETUP}

We start our discussion with introducing a formalism in equilibrium statistical mechanics that allows us to perform the coarse graining of generic $n$-body interactions. We consider a system composed by $N$ particles in $d$ spatial dimensions confined in a box of side $L$ and volume $V=L^{d}$. To keep the presentation simple, we indicate with $\left[r_{i}\right]$ or $\left(r_{i}\right)$ a generic particle configuration $\left(r_{1}, \ldots, r_{N}\right)$ where $r_{i}$ is a $d$-dimensional vector representing the position of the particle $i$. For the sake of completeness, we consider a Hamiltonian system composed of classical particles the degrees of freedom of which are canonical coordinates and conjugated momenta. In the next section, we will apply the formalism for computing configurational integrals in the case of active systems, and thus we will neglect generalized momenta.

Denoting $p_{i}$ the momentum of the particle $i$, we assume that the mechanical properties of the system are fully specified through the Hamiltonian function $H[p, r]$ :

$$
H\left[p_{i}, r_{i}\right]=\sum_{i=1}^{N} \frac{p_{i}^{2}}{2 m}+\mathcal{H}[r]
$$

where the configurational part $\mathcal{H}[r]$ takes into account onebody, two-body, and $k$-body interactions, with $k \geqslant 3$. The configurational energy is

$$
\begin{aligned}
\mathcal{H}\left[r_{i}\right]= & \sum_{i=1}^{N} \phi_{1}\left(r_{i}\right)+\frac{1}{2} \sum_{i, j}^{1, N} \phi_{2}\left(r_{i}, r_{j}\right) \\
& +\sum_{k \geqslant 3}^{N} \phi_{3}\left(r_{i}, \ldots, r_{k}\right) .
\end{aligned}
$$

Thermodynamics is obtained through the computation of the partition function $Z$ [39]:

$$
\begin{aligned}
Z & =\int \prod_{i} \frac{d r_{i}}{\lambda^{d N}} e^{-\beta H\left[r_{i}\right]+\beta \mu N}, \\
f(\beta, \mu) & =-\lim _{N, V \rightarrow \infty} \frac{1}{\beta V} \ln Z
\end{aligned}
$$

with $f(\beta)$ the free energy density. In Eq. (3), the thermodynamic limit $N, V \rightarrow \infty$ is performed maintaining fixed the mean density $\rho=N / V$. We have introduced the thermal wavelength $\lambda=\sqrt{\frac{h^{2}}{2 \pi m k_{B} T}}$. $\beta$ is the inverse temperature, i.e., $\beta=1 / k_{B} T ; h$ is the Planck constant; and $\mu$ is the chemical potential. Working in natural units, one has $k_{B}=1=h$ and thus $\beta=T^{-1}$.

For studying the behavior of the system on large scales, we perform a coarse graining based on the local-density field $\psi(r)$ :

$$
\psi(r)=\sum_{i} \delta\left(r-r_{i}\right)
$$

Using standard manipulations [27,40], one can enforce the field $\psi(r)$ into Eqs. (3) through a delta functional

$$
\int \mathcal{D} \psi(r) \delta\left[\psi(r)-\sum_{i} \delta\left(r-r_{i}\right)\right]=1
$$

and thus we can write

$$
Z=\int \mathcal{D} \psi(r) \mathcal{D} \hat{\psi}(r) e^{-G[\hat{\psi}, \psi]}
$$


where the auxiliary field $\hat{\psi}$ has been introduced for representing the delta functional introduced in Eq. (5). The details of the computation are provided in Appendix A. The functional $G$ takes the form

$$
\begin{aligned}
-G & \equiv-S[\psi]+\int d r[\hat{\psi}(r)-b(r)] \psi(r)+N \ln z, \\
z & \equiv \int \frac{d r}{\lambda^{d}} e^{-\hat{\psi}(r)},
\end{aligned}
$$

with

$$
\begin{aligned}
b(r) & \equiv \beta \phi_{1}(r)-\beta \mu, \\
S[\psi] & \equiv \frac{1}{2} \int d r d r^{\prime} \psi(r) \Delta^{-1}\left(r, r^{\prime}\right) \psi\left(r^{\prime}\right)+V[\psi], \\
\Delta^{-1} & \equiv \beta \phi_{2}\left(r, r^{\prime}\right), \\
V & \equiv \sum_{k \geqslant 3} \frac{1}{k !} \int d r_{1} \ldots d r_{k} \beta \phi_{k}\left(r_{1}, \ldots, r_{k}\right) \psi\left(r_{1}\right) \ldots \psi\left(r_{k}\right) .
\end{aligned}
$$

Performing a shift to the field $\hat{\psi}-b \rightarrow \hat{\psi}$, the thermodynamics can be recast in the following form:

$$
\begin{aligned}
e^{W[\hat{\psi}]} & \equiv \mathcal{N}^{-1} \int \mathcal{D} \psi e^{-S[\psi]+\int d r \hat{\psi}(r) \psi(r)}, \\
Z & =\mathcal{N} \int \mathcal{D} \hat{\psi} \mathcal{D} \psi e^{W[\hat{\psi}]+N \ln \int \frac{d r}{\lambda} e^{\hat{\psi}(r)-b(r)},} \\
\mathcal{N} & \equiv \int \mathcal{D} \psi e^{-S[\psi]} .
\end{aligned}
$$

As one can appreciate, the auxiliary field $\hat{\psi}$ in Eq. (9) plays the role of external source in a quantum field theory [40]. It is worth noting that $W[\hat{\psi}] \sim O(N)$. According to the definition of $W[\hat{\psi}]$, the $n$-point correlation function of the theory can be generated through functional differentiation. For instance, we have

$$
\begin{aligned}
\frac{\delta W}{\delta \hat{\psi}(r)} & =\langle\psi(r)\rangle, \\
\frac{\delta^{2} W}{\delta \hat{\psi}(r) \delta \hat{\psi}(s)} & =\langle\psi(r) \psi(s)\rangle_{C}
\end{aligned}
$$

where the average is defined as

$$
\langle O\rangle \equiv \frac{\int \mathcal{D} \psi e^{-S[\psi]+\int d r \psi(r) \hat{\psi}(r)} O}{\int \mathcal{D} \psi e^{-S[\psi]+\int d r \psi(r) \hat{\psi}(r)}}
$$

and $\langle\ldots\rangle_{C}$ indicates a connected correlation function.

\section{A. Stationary points, mean-field approximation, and fluctuations around the mean-field solution}

It is well known that mean-field theories neglect fluctuations [41]. In particular, in a mean-field approximation, one usually replaces the value of the order parameter in a given point of the space with its mean value in the same point, i.e., $\psi(r) \rightarrow\langle\psi(r)\rangle$ and $\left\langle\psi(r) \psi\left(r^{\prime}\right)\right\rangle \rightarrow\langle\psi(r)\rangle\left\langle\psi\left(r^{\prime}\right)\right\rangle$. The latter replacement holds whenever $\left\langle[\psi(r)-\langle\psi(r)\rangle]^{2}\right\rangle \sim$ $V^{-1 / 2} \sim N^{-1 / 2}$ that vanishes in the thermodynamic limit.
Through Eqs. (9) we define the functional $F[\hat{\psi}]$ :

$$
F[\hat{\psi}]=W[\hat{\psi}]+N \ln \int \frac{d r}{\lambda^{d}} e^{-\hat{\psi}(r)+b(r)} .
$$

Since the exponent in the functional integral is of order $N$, it makes sense to perform a saddle-point approximation for evaluating $Z$ and then compute systematically the stability of the stationary point $\hat{\psi}_{\text {SP }}$ against fluctuations. Considering field configurations $\hat{\psi}=\hat{\psi}_{\mathrm{SP}}+\Delta \hat{\psi}$, we can thus write

$$
\begin{aligned}
F[\hat{\psi}] & =F\left[\hat{\psi}_{\mathrm{SP}}\right]+F[\Delta \hat{\psi}], \\
F[\Delta \hat{\psi}] & \equiv \frac{1}{2} \int d r d s \Delta \hat{\psi}(r) \mathcal{G}(r, s) \Delta \hat{\psi}(s)
\end{aligned}
$$

where the kernel in Eqs. (13) is

$$
\mathcal{G}(r, s)=\left.\frac{\delta^{2} F}{\delta \hat{\psi}(r) \delta \hat{\psi}(s)}\right|_{\mathrm{SP}}
$$

and we have used the fact that

$$
\left.\frac{\delta F}{\delta \hat{\psi}(r)}\right|_{\mathrm{SP}}=0 \text {. }
$$

In terms of the generating functional $W$, we can define

$$
\begin{aligned}
\rho(r) & =\left.\frac{\delta W}{\delta \hat{\psi}(r)}\right|_{\mathrm{SP}}, \\
G(r, s) & =\left.\frac{\delta^{2} W}{\delta \hat{\psi}(r) \delta \hat{\psi}(s)}\right|_{\mathrm{SP}} .
\end{aligned}
$$

Neglecting terms that scale with $1 / N$, we can rewrite the partition function that is factorized as follows:

$$
\begin{aligned}
Z & =Z_{\mathrm{SP}} Z_{\text {fluct }}, \\
\ln Z_{\mathrm{SP}} & \equiv-F\left[\hat{\psi}_{\mathrm{SP}}\right], \\
Z_{\text {fluct }} & \equiv \int \mathcal{D} \varphi e^{-\int d r d s \varphi(r) \mathcal{G}(r, s) \varphi(s)}, \\
\mathcal{G}(r, s) & =G^{-1}(r, s)-\int d z G^{-1}(z, r) \rho(z) G^{-1}(z, s)
\end{aligned}
$$

where we have performed the change of variable $\Delta \hat{\psi}(r)=$ i $\int d s G^{-1}(r, s) \varphi(s)$ (computation details are provided in Appendix B). In Eqs. (17), $\mathcal{G}$ is the full propagator of the theory that takes contribution from both the two-body potential $\phi_{2}$ and the $k$-body potentials $\phi_{k}$.

Using the saddle-point relation Eqs. (15), we can write

$$
\rho(r)=\frac{N e^{-\hat{\psi}(r)+b(r)}}{z},
$$

obtaining in this way the following expression for the thermodynamics in the mean-field approximation:

$$
\begin{aligned}
Z_{\mathrm{SP}} & =e^{-F[\rho(r)]}, \\
f_{\mathrm{SP}}(\beta) & =\lim _{V, N \rightarrow \infty} \frac{F[\rho(r)]}{\beta V} .
\end{aligned}
$$

\section{MICROSCOPIC MODEL OF ACTIVE PARTICLES}

As a microscopic model, we consider active particles that are self-propelled through Ornstein-Uhlenbeck processes, i.e., active Ornstein-Uhlenbeck particles (AOUPs) [22,24,42-45]. 
The equations of motion for AOUPs are $[16,22,23,42,46]$

$$
\begin{aligned}
\dot{\mathbf{x}}_{i} & =\mu\left(\mathbf{f}_{i}^{a}+\mathbf{f}_{i}^{m}+\mathbf{f}_{i}^{\text {ext }}\right), \\
\tau \dot{\mathbf{f}}_{i}^{a} & =-\mathbf{f}_{i}^{a}+\boldsymbol{\eta}_{i} .
\end{aligned}
$$

Here we indicate with $\mathbf{x}_{i}$ a vector in $d$ spatial dimensions that determines the position of the active particle $i$. The selfpropulsive force is $\mathbf{f}_{i}^{a}$ characterized by a persistence time $\tau$. The force on particle $i$ resulting from the interactions with other particles is $\mathbf{f}_{i}^{m}$. The term $\mathbf{f}_{i}^{\text {ext }}$ represents forces due to external conservative fields. Finally, $\mu$ is the mobility. The noise $\eta_{i}$ satisfies $\left\langle\eta_{i}^{\alpha}\right\rangle=0$ and $\left\langle\eta_{i}^{\alpha}(t) \eta_{j}^{\beta}(s)=2 T \mu \delta_{i j} \delta^{\alpha \beta} \delta(t-s)\right.$, where greek symbols indicate Cartesian components. According to Eqs. (20), the self-propulsion is exponentially correlated in time; in particular, one has $\left\langle f_{i}^{a, \alpha}(t) f_{j}^{a, \beta}(s)\right\rangle=$ $\frac{2 T \mu}{\tau} e^{-|t-s| / \tau}[17]$.

Let us introduce the steady-state distribution $P_{s s}\left(\mathbf{x}_{i}\right) \equiv$ $P_{s s}\left(\mathbf{x}_{1}, \ldots, \mathbf{x}_{N}\right)=\lim _{t \rightarrow \infty} P_{s s}\left(\mathbf{x}_{1}(t), \ldots, \mathbf{x}_{N}(t)\right)$. Adopting the unified colored noise approximation [25,26], it has been shown $[22,43-45,47]$ that the approximate solution for $P_{s s}$ takes the form

$$
\begin{aligned}
P_{s s}\left(\mathbf{x}_{i}\right) & =Z_{\beta}^{-1} \exp \left(-\beta H_{\mathrm{UCN}}\left[\mathbf{x}_{i}\right]\right), \\
H_{\mathrm{UCN}}\left[\mathbf{x}_{i}\right] & =H_{0}\left[\mathbf{x}_{i}\right]+H_{1}\left[\mathbf{x}_{i}\right]+H_{2}\left[\mathbf{x}_{i}\right], \\
H_{0}\left[\mathbf{x}_{i}\right] & \equiv \frac{1}{2} \sum_{i, j} \phi_{2}\left(\mathbf{x}_{i}, \mathbf{x}_{j}\right)+\sum_{i} \phi_{1}\left(\mathbf{x}_{i}\right), \\
H_{1}\left[\mathbf{x}_{i}\right] & \equiv \frac{\tau}{2} \sum_{i}\left(\nabla_{\mathbf{x}_{i}} H_{0}\right)^{2}, \\
H_{2}\left[\mathbf{x}_{i}\right] & \equiv-\beta^{-1} \ln \operatorname{det} \mathbf{M}, \\
\mathbf{M} & \equiv M_{i j}^{\alpha \gamma}=\delta_{i j}^{\alpha \gamma}+\tau \frac{\partial^{2} H_{0}}{\partial x_{i}^{\alpha} \partial x_{j}^{\gamma}}
\end{aligned}
$$

with $Z_{\beta}$ fixed by the normalization condition $\int \prod_{i} d \mathbf{x}_{i} P_{s s}\left(\mathbf{x}_{i}\right)=1$. The Hamiltonian $H_{0}$ is responsible for both the mechanical interactions and the interactions with external fields, i.e., $\mathbf{f}_{i}^{m}+\mathbf{f}_{i}^{\text {ext }}=-\nabla_{\mathbf{x}_{i}} H_{0}\left[\mathbf{x}_{i}\right]$. As one can see, $P_{s s}\left(\mathbf{x}_{i}\right)$ takes the form of an equilibrium distribution where the Hamiltonian $H_{0}$ is replaced by an effective one that we named $H_{\mathrm{UCN}}\left[\mathbf{x}_{i}\right]$. In this way, the structural properties of the system on large scale can be computed through an equilibrium statistical mechanics theory based on $H_{\mathrm{UCN}}\left[\mathbf{x}_{i}\right]$. In the present paper, we will apply the theoretical machinery introduced in the previous section for coarse graining the equilibrium-like model [48].

The effective Hamiltonian $H_{\mathrm{UCN}}\left[\mathbf{x}_{i}\right]$ is composed of three contributions. The term $H_{0}$ is the mechanical energy of the system in equilibrium. The terms $H_{1}$ and $H_{2}$ introduce nonlocal many-body interactions among particles that disappear in the limit $\tau \rightarrow 0$. The computation of $\operatorname{det} M$ in $d$ spatial dimensions requires the diagonalization of a $d N \times d N$ matrix. Moreover, Eq. (21) requires that $\mathbf{M}$ must be a positive definite matrix. Here we will consider a small $\tau$ expansion based on the approximation $\operatorname{det}\left(\delta_{i j}^{\alpha \gamma}+\tau D_{i j}^{\alpha \gamma}\right)=1+\tau \operatorname{Tr} D_{i j}^{\alpha \gamma}+o\left(\tau^{2}\right)$ (see Appendix E), with $D_{i j}^{\alpha \gamma}$ the Hessian matrix. It is worth noting that the small $\tau$ breaks when the Hessian develops negative eigenvalues of order $\tau^{-1}$. In this paper, we will restrict our computation in cases where $D_{i j}^{\alpha \gamma}$ is positive definite.

\section{ONE-BODY INTERACTIONS}

Since active particles break the fluctuation-dissipation theorem at the single-particle level [49-51], they show intriguing nonequilibrium phenomena even at the level of gas of noninteracting particles. In particular, when active particles are confined by a container or immersed into a confining potential, i.e., a central field that tends to confine particles into a region of space, the steady-state distribution strongly deviates from the Boltzmann distribution, showing a double-peaked structure at high persistence time [5,52-54]. The double peaks signal the accumulation of active particles at the boundary of the confining potential instead in the center, i.e., where the potential is zero. This effect is due to the fact that active particles remain trapped in regions of space where the external field exerts forces that balance the self-propulsion force rather than in a region where the potential is zero. Accumulation at the boundaries has been also observed in experiments [55].

For making the notation simplest, without loss of generality, we consider a one-dimensional problem. We indicate with $x$ the coordinate of the active particle. Indicating with the prime the derivative with respect to $x$, we identify with $x_{0}$ the solution of $\left.H_{\text {eff }}(x)^{\prime}\right|_{x=x_{0}}=0$. In this section, we will see that this nonequilibrium phenomenon can be interpreted as a spontaneous symmetry breaking in the effective Hamiltonian $H_{\text {eff }}(x)$. For $\tau=0$, one recovers the usual equilibrium phenomenology. In particular, while a system in equilibrium develops condensation at the bottom of the external potential, i.e., where $H^{\prime}\left(x_{0}=0\right)=0$, active particles condensate away from the bottom in regions where $H_{\text {eff }}^{\prime}\left(x_{0}\right)=0$. This can be rationalized through our formalism in terms of an effective potential that develops a double-well structure as soon as $\tau>0$. The condensation away from the center of the trapping potential can be thus interpreted as a spontaneous symmetry breaking in an effective equilibrium picture. As a benchmark for the formalism, in Appendix $\mathrm{C}$ we report the case of gas in equilibrium in one spatial dimension embedded into an external potential where no accumulation at the boundaries takes place and localization appears at the bottom of the potential.

\section{A. Gas of active particles in external potentials}

We consider a gas of active particles embedded into an external potential $A(x)$ in one spatial dimension. As we showed in the previous section, the steady-state properties of the system can be obtained through the computation of the following partition function:

$$
Z_{\beta}=\int \prod_{i} d x_{i} e^{-\beta H_{\mathrm{eff}}\left[x_{i}\right]}
$$

where the Hamiltonian $H_{\mathrm{eff}}\left[x_{i}\right]$ is

$$
\begin{aligned}
H_{\mathrm{eff}}\left[x_{i}\right]= & \sum_{i=1}^{N} A\left(x_{i}\right)+\frac{\tau}{2} \sum_{i=1}^{N}\left[A^{\prime}\left(x_{i}\right)\right]^{2} \\
& -\beta^{-1} \sum_{i=1}^{N} \ln \left[1+\tau A^{\prime \prime}\left(x_{i}\right)\right]
\end{aligned}
$$


and we can thus write

$$
\begin{aligned}
H_{\text {eff }}\left[x_{i}\right] & =\sum_{i=1}^{N} B\left(x_{i}\right), \\
B\left(x_{i}\right) & \equiv A\left(x_{i}\right)+\frac{\tau}{2} A^{\prime}\left(x_{i}\right)^{2}-\beta^{-1} \ln \left[1+A^{\prime \prime}\left(x_{i}\right)\right] .
\end{aligned}
$$

In terms of the density field $\psi(x)=\sum_{i} \delta\left(x-x_{i}\right)$, the partition function is

$$
\begin{aligned}
Z_{\beta} & =\int \mathcal{D} \psi(x) e^{-\beta G[\psi]}, \\
G[\psi] & \equiv \int d x \psi(x)[\beta B(x)+\ln \psi(x)] .
\end{aligned}
$$

We can approximate the integral using the saddle-point approximation

$$
Z_{\beta} \sim e^{-\beta G[n]}
$$

where we have indicated with $n(x)$ the field configuration that solves

$$
\left.\frac{\delta G}{\delta \psi(x)}\right|_{\psi=n}=0 .
$$

The steady-state density profile reads

$$
n(x)=c e^{-\beta B(x)} .
$$

At small temperatures the density profile is dominated by the minima $x_{0}$ of $B(x)$ and thus $n(x)$ is concentrated around those minima, i.e., $n(x) \sim \sum_{\kappa} \delta\left(x-x_{0}^{\kappa}\right)$, where $\kappa$ labels the solutions of

$$
\left.\frac{d B}{d x}\right|_{x=x_{0}^{\kappa}}=0,\left.\quad \frac{d^{2} B}{d x^{2}}\right|_{x=x_{0}^{\kappa}} \geqslant 0 .
$$

We specialize our computation in the case of harmonic and anharmonic trapping, the latter due to a quartic confining potential.

\section{B. Soft confining potentials in active matter}

Self-propulsion naturally introduces a typical scale for the forces, i.e., the self-propulsion force $f_{s}$, that competes with the other force fields interacting with the active particles. Due to this fact, even smooth potential fields can give rise to dramatic confining effects. Now we consider a generic confining potential of the form

$$
A(x)=\frac{x^{2 \alpha}}{2 \alpha}
$$

with the exponent $\alpha=1,2, \ldots$ In this way, for $\alpha=1$, one recovers the harmonic trap. The steepest confining potentials are obtained for $\alpha>2$. In particular, we will focus our attention on $\alpha=2$. The effective potential $B(x)$ reads

$$
B(x)=\frac{x^{2 \alpha}}{2 \alpha}+\frac{\tau}{2} x^{4 \alpha-2}-\beta^{-1} \ln \left[1+\tau(2 \alpha-1) x^{2 \alpha-2}\right]
$$

and the derivative is

$$
\begin{aligned}
B^{\prime}(x)= & x^{2 \alpha-1}+\frac{\tau}{2}(4 \alpha-2) x^{4 \alpha-3} \\
& -\frac{\tau}{\beta} \frac{(2 \alpha-1)(2 \alpha-2) x^{2 \alpha-3}}{1+\tau(2 \alpha-1) x^{2 \alpha-2}} .
\end{aligned}
$$

\section{Harmonic trapping of AOUPs}

The harmonic trapping is recovered for $\alpha=1$. As it has been proved experimentally and verified in simulations [51], harmonic potentials lead to a generalization of the equipartition theorem. This is due to the fact that the effective energy takes the simple form

$$
B(x)=\frac{1+\tau}{2} x^{2}-\beta^{-1} \ln (1+\tau)
$$

that is the energy of an harmonic oscillator where the natural frequency $\omega_{0}$ results shifted from $\omega_{0}=1$ to $1+\tau$. This result means that no accumulation at the boundaries occurs in the case of AOUPs trapped through a harmonic potential.

\section{Anharmonic trapping of AOUPs}

Now we are going to show that the effective equilibrium picture reproduces the accumulations of active particles at the boundary of a confining container. For the sake of simplicity, we consider the case $\alpha=2$, i.e., a soft anharmonic confining potential instead of a container with hard boundaries. $B(x)$ reads

$$
B(x)=\frac{x^{4}}{4}+\frac{\tau}{2} x^{6}-\beta^{-1} \ln \left[1+3 \tau x^{2}\right] .
$$

Now a density profile $n(x)$ that is peaked around $x_{0}=0$ turns out to be unstable. To rationalize that, we compute the second derivative of $B(x)$ :

$$
B^{\prime \prime}(x)=3 x^{2}+15 \tau x^{4}+\frac{36 x^{2} \tau^{2}}{\beta\left(1+3 \tau x^{2}\right)^{2}}-\frac{6 \tau}{\beta\left(1+3 \tau x^{2}\right)} .
$$

As one can immediately check, the configuration $x_{0}=0$ turns out to be unstable since $B^{\prime \prime}(0)=-\frac{6 \tau}{\beta}<0$; i.e., as soon as $\tau \neq 0, x_{0}=0$ is not a minimum anymore. In terms of $B(x)$, the emerging phenomenology can be interpreted as a spontaneous symmetry breaking of the symmetry $x \rightarrow-x$ driven by motility, having $\tau$ as a control parameter instead of temperature [40], i.e., $T_{\text {eff }} \sim \tau^{-1}$. Under this perspective, $\tau$ drives a phase transition from a more symmetric warm phase, where particles are localized around $x_{0}=0$, to a less symmetric cold phase, where particles are localized away from $x_{0}=0$. To prove that we consider the small $\tau$ expansion of Eq. (34):

$$
B(x)=-3 \tau x^{2}+\left(\frac{1}{4}+\frac{9 \tau^{2}}{2}\right) x^{4}+o\left(x^{6}\right) .
$$

As one can see, if we think of the potential $A(x)$ in terms of a Landau theory, the potential can be interpreted as a mean-field theory $\frac{a}{2} x^{2}+\frac{x^{4}}{4}$ at the critical point, i.e., $a=0$. Now, for $\tau>$ 0 , the system is described by Landau energy that is given by Eq. (36). Looking at the coefficient of the $x^{2}$ term as a mass it turns to be unphysical since it is negative. This means that the original vacuum of the theory, i.e., $x_{0}=0$, is not a minimum anymore and thus $B(x)$ spontaneously breaks the symmetry $x \rightarrow-x$ that was satisfied by $A(x)$. Now the new stable configuration of lower energy is $x_{0}^{1,2}= \pm \sqrt{\frac{6 \tau}{1+18 \tau^{2}}} \equiv \pm v$. Density profiles at zero temperature take the form $n(x) \sim \delta(x-$ $\left.x_{0}^{1}\right)+\delta\left(x-x_{0}^{2}\right)=\delta(x-v)+\delta(x+v)$. A finite but small temperature $T=1 / \beta$ introduces a finite variance in the two 


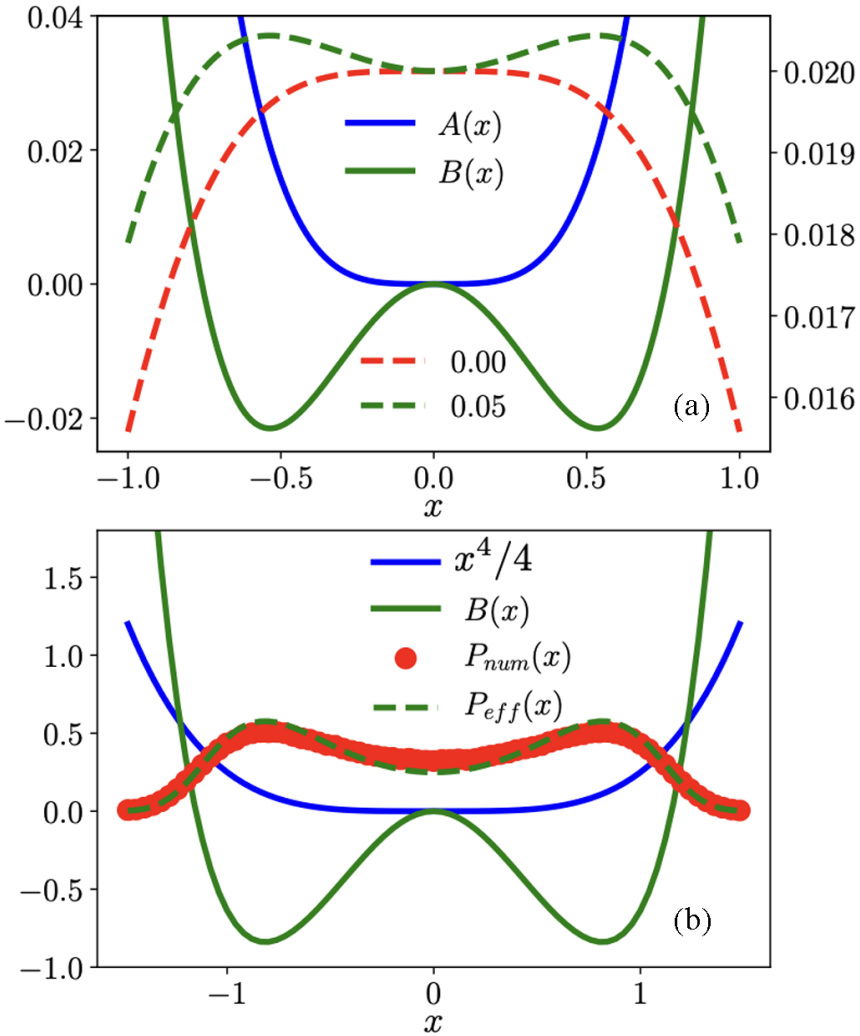

FIG. 1. (a) A gas of active particles embedded into an anharmonic trap in one spatial dimension, $A(x)=\frac{x^{4}}{4}$ (solid blue curve). $B(x)$ is the effective potential felt by a particle when $\tau=0.05$. Dashed curves refer to the probability distribution functions. $B(x)$ breaks the symmetry $x \rightarrow-x$. (b) Comparison between numerical simulations of the actual nonequilibrium dynamics (red circles) and effective theory (dashed green curve). The solid blue curve is the anharmonic trapping potential; the solid green curve indicates the effective Hamiltonian describing the system. The simulation parameters are $\tau=1, \mu=1$, and $T=1$.

distributions that remain peaked around $\pm v$. Expanding around the new minima $x=v+\delta$ at the linear order in $\tau$ one has

$$
B(\delta x)=6 \delta x^{2} \tau+\frac{\delta x^{4}}{4}+\sqrt{6} \delta x^{3} \sqrt{\tau}+o\left(\tau^{2}\right) .
$$

As one can see, fluctuations $\delta x$ around the minimum $v$ acquire a mass that is linear in $\tau$. The emerging phenomenology is shown in the upper panel of Fig. 1 where the solid blue line is the central potential $A(x)$ with $\alpha=2$ and the solid green line is $B(x)$ that develops two symmetric minima that break the symmetry $x \rightarrow-x$. The dashed lines are the distribution function $n(x) \sim \exp [-B(x)]$ for $\tau=0$ and 0.05 (dashed red and dashed green, respectively). As one can appreciate, for $\tau=0$, one has the peak of the distribution at $x_{0}=0$. For $\tau=$ 0.05 , the distribution becomes double peaked.

Moreover, the theoretical picture provides also approximation schemes for obtaining quantitative predictions. We thus performed numerical simulations of a gas of AOUPs embedded into the potential $A(x)$. Through the numerical integration of the actual dynamics given by Eqs. (20), we sampled steady-state trajectories for computing numerically
$P_{\text {num }}(x)$ (details are given in Appendix H). The comparison between $P_{\text {num }}(x)$ and $P_{\text {eff }}(x)$ obtained from UCN is shown in the lower panel of Fig. 1. The red circles represent the histogram $P(x)$ computed from numerical data, considering $N=4000$ independent runners. $P_{\text {eff }}(x)$ has been computed considering $P_{\text {eff }}(x)=\mathcal{N} e^{-B(x) / T}$ with $\mathcal{N}$ a normalization constant that guaranties $\int d x P_{\mathrm{eff}}(x)=1$. Here, the computation has been performed considering the expression of $B(x)$ given in Eq. (34), i.e., without performing any small $\tau$ expansion.

\section{MANY-BODY INTERACTIONS: MEAN-FIELD THEORY}

Now we are going to consider the case of two-body interactions. With this aim, we perform the coarse-grained computation of Eq. (21) in the absence of external fields, i.e., $\phi_{1}=0$. For the sake of simplicity we indicate the two-body potential $\phi_{2}(\mathbf{x}, \mathbf{y})=\phi(\mathbf{x}, \mathbf{y})$. Moreover, for the computation of the determinant that appears in Eq. (21), we recover to a small $\tau$ expansion. In terms of the density field $\rho(\mathbf{x})$, using the theoretical framework introduced in Eq. (II), we can write the following mean-field model (details of the computation are provided in Appendix E):

$$
\begin{aligned}
f(\beta)= & -\lim _{N, V \rightarrow \infty} \frac{1}{\beta V} \ln Z, \\
Z= & \int \mathcal{D} \hat{\psi}(\mathbf{x}) \mathcal{D} \psi(\mathbf{x}) e^{-G[\hat{\psi}, \psi]} \sim e^{-S_{\mathrm{eff}}[\rho(x)]}, \\
-S_{\mathrm{eff}} \equiv & \frac{1}{2} \int d \mathbf{x} d \mathbf{y} \rho(\mathbf{x}) A(\mathbf{x}, \mathbf{y}) \rho(\mathbf{y}) \\
& +\frac{\tau}{2} \int d \mathbf{x} d \mathbf{y} d \mathbf{z} \rho(\mathbf{x}) \rho(\mathbf{y}) \rho(\mathbf{z}) B(\mathbf{x}, \mathbf{y}, \mathbf{z}) \\
& +\int d \mathbf{x} \rho(\mathbf{x})\left[1+\ln \left(\frac{1-\rho(\mathbf{x})}{\rho(\mathbf{x})}\right)\right], \\
A(\mathbf{x}, \mathbf{y}) \equiv & \phi(\mathbf{x}, \mathbf{y})-2 \tau T \partial_{\mathbf{x}, \mathbf{y}}^{2} \phi(\mathbf{x}, \mathbf{y}), \\
B(\mathbf{x}, \mathbf{y}, \mathbf{z}) \equiv & \partial_{\mathbf{z}} \phi(\mathbf{z}, \mathbf{x}) \partial_{\mathbf{z}} \phi(\mathbf{z}, \mathbf{y}) .
\end{aligned}
$$

As one can see, the action $S_{\text {eff }}$ results from three contributions: (i) a two-body interaction with kernel $A(\mathbf{x}, \mathbf{y})$, (ii) a three-body interaction with kernel $B(\mathbf{x}, \mathbf{y}, \mathbf{z})$, and (iii) an entropic term that has a combinatorial origin [56,57]. It is worth noting that, as we will see in detail in the next sections, the two-body interaction, that is responsible also for the mass term in the corresponding field theory, can change the sign because of the competition between the original interaction $\phi$ and quadratic terms originated by the small $\tau$ expansion of det M. When it is different from zero, the three-body interaction developed by the effective equilibrium picture introduces an asymmetry between $\rho(\mathbf{x})$ and $1-\rho(\mathbf{x})$. It is worth noting that, considering a simple van der Waals description of the liquid-gas coexistence, there is a natural asymmetry between the coexistent phases. However, around the critical point of the gas-liquid phase transition, it is possible to perform an opportune Landau-Ginzburg expansion in even powers of the order parameter. We will see that $B$ introduces an odd power in that expansion. 


\section{A. Central pair potentials}

Equation (38) defines the thermodynamic of a wide class of scalar active systems. In order to make quantitative progress, we have to define the form of the two-body interaction. Let us focus our attention on the case of central pair potentials that take the form $\phi\left(\mathbf{x}_{i}, \mathbf{x}_{j}\right)=\phi\left(\left|\mathbf{x}_{i}-\mathbf{x}_{j}\right|\right)=\phi\left(r_{i j}\right)$ where we have defined $r_{i j} \equiv\left|\mathbf{x}_{i}-\mathbf{x}_{j}\right|$. Indicating with the prime the derivative with respect to $r$, the diagonal part of the Hessian matrix reads

$$
\begin{aligned}
H_{i i}^{\alpha \alpha}= & \sum_{l}\left\{\frac{\phi^{\prime \prime}\left(r_{l i}\right)}{r_{l i}^{2}}\left(x_{l}^{\alpha}-x_{i}^{\alpha}\right)^{2}\right. \\
& \left.+\phi^{\prime}\left(r_{l i}\right)\left[\frac{1}{r_{l i}}-\frac{\left(x_{l}^{\alpha}-x_{i}^{\alpha}\right)^{2}}{r_{l i}^{3}}\right]\right\} .
\end{aligned}
$$

In terms of the local-density field $\psi(\mathbf{x})$ we can thus rewrite $\mathrm{H}_{2}$ as follows:

$$
\begin{aligned}
H_{2}[\psi] & =-\tau T \int d \mathbf{x} d \mathbf{y} \psi(\mathbf{x}) f(|\mathbf{x}-\mathbf{y}|) \psi(\mathbf{y}), \\
f(r) & \equiv \phi^{\prime \prime}(r)+\frac{\phi^{\prime}(r)}{r}(d-1)
\end{aligned}
$$

The contribution of $H_{1}$ becomes

$$
\begin{aligned}
H_{1}[\psi]= & \frac{\tau}{2} \int d \mathbf{x} d \mathbf{y} d \mathbf{z} B(\mathbf{x}-\mathbf{y}, \mathbf{x}-\mathbf{z}) \phi^{\prime}\left(r_{x z}\right) \\
& \times \psi(\mathbf{x}) \psi(\mathbf{y}) \psi(\mathbf{z}) \\
B(\mathbf{x}-\mathbf{y}, \mathbf{x}-\mathbf{z}) \equiv & \frac{(\mathbf{x}-\mathbf{y}) \cdot(\mathbf{x}-\mathbf{z})}{r_{x y} r_{x z}}
\end{aligned}
$$

At the saddle point we have $\langle\psi(\mathbf{x})\rangle=\rho(\mathbf{x})$ and thus we can finally write the effective action for central potentials:

$$
\begin{aligned}
S_{\mathrm{eff}}[\rho]= & \int d \mathbf{x} d \mathbf{y} \rho(\mathbf{x}) A(|\mathbf{x}-\mathbf{y}|) \rho(\mathbf{y}) \\
& +\frac{\tau}{2} \int d \mathbf{x} d \mathbf{y} d \mathbf{z} B(|\mathbf{x}-\mathbf{y}|,|\mathbf{x}-\mathbf{z}|) \rho(\mathbf{x}) \rho(\mathbf{y}) \rho(\mathbf{z}) \\
& +\int d \mathbf{x} \rho(\mathbf{x})\left[1+\ln \left(\frac{1-\rho(\mathbf{x})}{\rho(\mathbf{x})}\right)\right]
\end{aligned}
$$

where the functions $A(r)$ and $B(r, s)$ now read

$$
\begin{aligned}
A(r) & =\phi(r)-\tau T f(r), \\
B(r, s) & =\frac{\mathbf{r} \cdot \mathbf{s}}{r s} \phi^{\prime}(r) \phi^{\prime}(s) .
\end{aligned}
$$

\section{B. Motility-induced phase separation}

The action $S_{\text {eff }}$ in Eq. (42) describes the phenomenology of MIPS [8,15,38,58-60], i.e., the ability of active systems to undergo a gas-liquid transition in the absence of any attractive force. The mechanism underlying MIPS is well understood [8]. It is based on the fact that active particles move slower where they are denser. As it has been shown in early works on AOUPs [22,24], the same crucial ingredient enters through the term $\mathrm{H}_{2}$ in Eq. (21) that plays the role of a local and diffusion coefficient [24]. In our picture, the dependency of $\mathrm{H}_{2}$ on density, and thus of the density-dependent diffusion, competes with the density dependency of $H_{0}$ and
$H_{1}$. As in other approaches in studying MIPS, also in this case the functional dependency of the diffusion coefficient can trigger long-wavelength instabilities that eventually lead to phase separation [17].

To rationalize that, let us consider the stability of a homogeneous density profile $\bar{\rho}$. As we will see in detail in the next sections, considering homogeneous solutions, at the saddle point we can write $S_{\text {eff }}[\bar{\rho}]=V g(\bar{\rho})$, where the function $g(\bar{\rho})$ defines the intensive Gibbs free energy. The stability of $\bar{\rho}$ that minimizes $g(\bar{\rho})$ depends on the sign of the second derivative of $g$ with respect to $\rho$ computed in $\bar{\rho}$. In particular, in the case of van der Waals theory, the negative sign of the coefficient $\rho^{2}$ ensures the possibility that a homogeneous density profile may become unstable in a certain region of the phase diagram [56]. If one neglects attractive interactions, the coefficient in the quadratic term is positive and thus there is no hope to observe a spinodal decomposition.

In the case of active particles, $A(r)$ takes two contributions. The first one is due to the two-body central potential $\phi(r)$. This is the standard term that one has in the equilibrium theory: it is attractive or repulsive depending on the type of potential. The second one is given by the function $f(r)$ and is linear in $\tau$, i.e., it disappears for $\tau=0$. The important thing is that the function $f(r)$ can be always seen as an attractive potential [42]. The intensity of the attraction is tuned by $\tau$. However, as we will discuss in the next section, in the case of repulsive potentials, there is a threshold value of $\tau$ for observing MIPS. Focusing our attention on the location of the critical point of MIPS, this fact implies a reentrant phase diagram [61,62]; i.e., at small enough self-propulsion velocities, MIPS disappears. This means that, in a certain region of the phase diagram, the system undergoes MIPS and then eventually goes back to the gas phase as $\tau$ becomes larger than the threshold value.

\section{MEAN-FIELD THEORY FOR SCALAR ACTIVE SYSTEMS}

In this section, we discuss the mean-field solutions of Eq. (42) assuming homogeneous density profiles. In this way, we describe the phase coexistence in active fluids through a van der Waals-like equation. We discuss the general case of active particles interacting via a central potential. We thus specialize our computation in both cases: repulsive potentials that give rise to MIPS and Lennard-Jones potentials. Since the effective equilibrium theory represented by Eq. (42) depends on spatial derivatives of the interacting potential $\phi(r)$, it is important that $\phi(r)$ is repulsive on short distances but also a smooth function, in order to have well-defined first and second derivatives with respect $r$.

With this aim, we consider the following central potential that results from two contributions: the first one represents a hard-core repulsion that provides well-defined excluded volume effects; the second one is a smooth function that ensures well-defined derivatives. We consider the following function:

$$
\phi(r)=\phi_{\mathrm{HS}}^{\sigma}+\phi_{\text {smooth }}(r)
$$

where $\sigma$ is the particle radius. The smooth part $\phi_{\text {smooth }}(r)$ is a continuous function of class $\mathcal{C}^{\infty}$, as it is shown in Fig. 2. We 


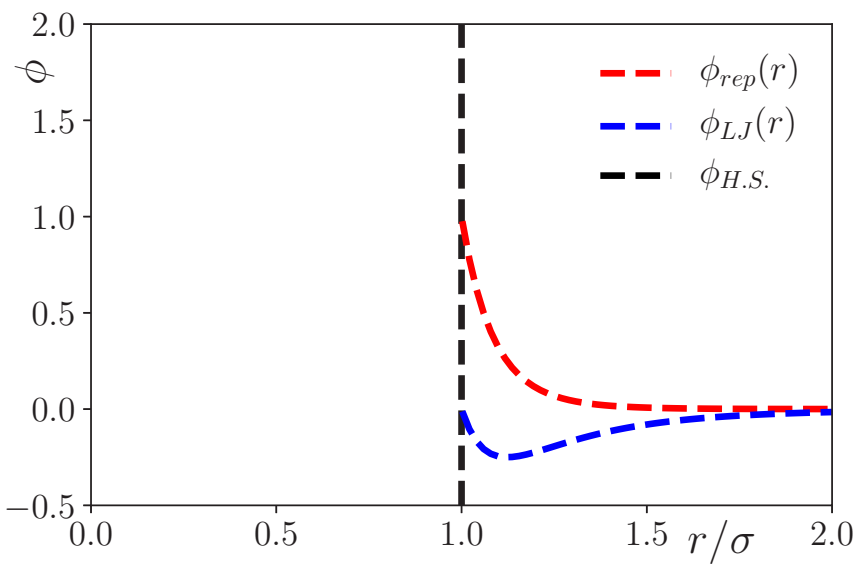

FIG. 2. Interaction potentials. We consider an interaction potential $\phi=\phi_{\mathrm{HS}}+\phi_{\text {smooth }}$ that is composed by a hard-core repulsion (dashed black line) and a smooth interaction $\phi_{\text {smooth }}$. For investigating MIPS, the smooth part consists in a purely repulsive potential (dashed red line). We also consider a Lennard-Jones potential (dashed blue line).

can thus write

$$
\phi_{\mathrm{HS}}^{\sigma}(r)=\left\{\begin{array}{cc}
0 & r>\sigma \\
\infty & r \leqslant \sigma
\end{array}\right.
$$

and

$$
\phi_{\text {smooth }}(r)=\left\{\begin{array}{cc}
0 & r \leqslant \sigma \\
\phi(r) & r>\sigma
\end{array} .\right.
$$

Homogeneous density profiles $\rho$ are described by the mean density of the system: $\rho(\mathbf{x})=\rho=N / V$. We can thus rewrite the partition function in Eq. (42) as follows:

$$
Z \sim e^{-V g(\rho)},
$$

where the free energy $g(\rho)$ reads

$$
\begin{aligned}
-g(\rho) & =\alpha(\tau, \beta) \rho^{2}+\frac{\tau b}{2} \rho^{3}+\rho\left[\ln \frac{1-\rho}{\rho}+1\right], \\
b & \equiv \int d \mathbf{x} d \mathbf{y} d \mathbf{z} \frac{(\mathbf{x}-\mathbf{y}) \cdot(\mathbf{x}-\mathbf{z})}{r_{x y} r_{x z}} \phi^{\prime}\left(r_{x y}\right) \phi^{\prime}\left(r_{x z}\right), \\
\alpha(\tau, \beta) & =\Omega(d) \int d r r^{d-1}[\beta \phi(r)-\tau f(r)], \\
f(r) & \equiv \phi^{\prime \prime}(r)+\frac{\phi^{\prime}(r)}{r}(d-1) .
\end{aligned}
$$

In the case of homogeneous density profiles due to spherical particles, the coefficient $b=0$ for symmetry reasons. The theory reduces to van der Waals model defined by the following free energy:

$$
-g(\rho)=\alpha(\tau, \beta) \rho^{2}+\rho\left[\ln \frac{1-\rho}{\rho}+1\right] .
$$

As one can see, Eq. (49) is precisely the van der Waals free energy where the effects of interaction and motility are reabsorbed into the coefficient $\alpha(\tau, \beta)$ [57]. Homogeneous density profiles turn out to be stable whenever $\alpha(\tau, \beta) \geqslant 0$. It is worth noting that, in the case of equilibrium systems, the coefficient is always positive in the case of purely repulsive potentials and thus there is no hope to observe spinodal decompositions at equilibrium without attractive forces.

\section{A. Repulsive potentials: MIPS critical point and inverse melting}

For a purely repulsive potential as in the case of $\phi_{\text {smooth }}(r)=\left(\frac{\sigma}{r}\right)^{12}$, the coefficient $\alpha$ reads

$$
\alpha(\tau, \beta)=\Omega_{d} \sigma^{d}\left[\frac{\beta}{12-d}-\frac{12 \tau}{\sigma^{2}}\right],
$$

with $\Omega_{d} \equiv \frac{2 \pi^{d / 2}}{\Gamma\left(\frac{d}{2}\right)}$ and $\Gamma(x)$ the Gamma function. As we said in the previous section, $\alpha$ is always positive for $\tau \rightarrow 0$, indicating that there is no way to observe spinodal decomposition in equilibrium systems. Because of the specific choice of the microscopic pair potential we have made, the space dimensions must be smaller than 12 . For $d<12$, the negative and positive values of $\alpha$ are bounded by the curve

$$
\beta_{\mathrm{th}}(\tau)=\frac{12(12-d)}{\sigma^{2}} \tau .
$$

We can write the pressure $P(\rho)$ as

$$
P(\rho)=\rho\left(\alpha(\beta, \tau) \rho+\frac{1}{\beta \rho(1-\rho)}\right) .
$$

Binodal lines can be computed considering the solution of $P(\rho)=P(1-\rho)$ [63]. The phase diagram can be obtained considering the solution of $\partial_{\rho} P=0$ that provides the coexistence curve $\tau(T, \rho)$. The computation in $d$ spatial dimensions and $\sigma=1$ brings us to

$$
\tau(T, \rho)=\frac{d T^{2}-2 \rho^{3}+4 \rho^{2}-2 \rho-12 T^{2}}{24(d-12)(\rho-1)^{2} \rho T} .
$$

The phase diagram in two spatial dimensions is shown in Fig. 3, left panel. The location of the critical point can be obtained considering the solutions of $\partial_{\rho} P=\partial_{\rho}^{2} P=0$ that individuate the point $\rho_{c}=\frac{1}{3}$ and $\tau_{c}(T)=\frac{27 d T^{2}-324 T^{2}-8}{96(d-12) T}$. As one can see, we obtain the same critical density of van der Waals theory.

We can also compute the critical temperature as a function of the correlation time of the noise:

$$
T_{c}(\tau)=\frac{16 \tau}{9} \pm \frac{2 \sqrt{2}}{9(d-12)} \sqrt{(d-12)\left[3+32(d-12) \tau^{2}\right]} .
$$

The behavior of $T_{c}(\tau)$ for $d=2$ is shown in the right panel of Fig. 3. As one can appreciate, the critical line as a function of $\tau$ is nonmonotonic, developing a reentrant behavior in the $T$ vs $\tau$ plane. It is worth noting that there is a threshold value $\tau_{\text {th }}$ of $\tau$ for having spinodal decomposition. Below $\tau_{\text {th }}$, i.e., for $\tau<\tau_{\text {th }}$, the system does not undergo a phase transition. Moreover, the critical line $T_{c}(\tau)$ develops a reentrant phase diagram. Meaning that, decreasing $T$ at fixed $\tau$, the system crosses a line of critical points at $T_{1}=T_{c}\left(\tau^{1}\right)$ and then, as $T$ decreases below $T_{2}=T_{c}\left(\tau^{2}\right) \leqslant T_{1}$, the system crosses again the line and it comes back to the original gas phase. The reentrant phase diagram is highlighted in the inset of Fig. 3, right panel. 

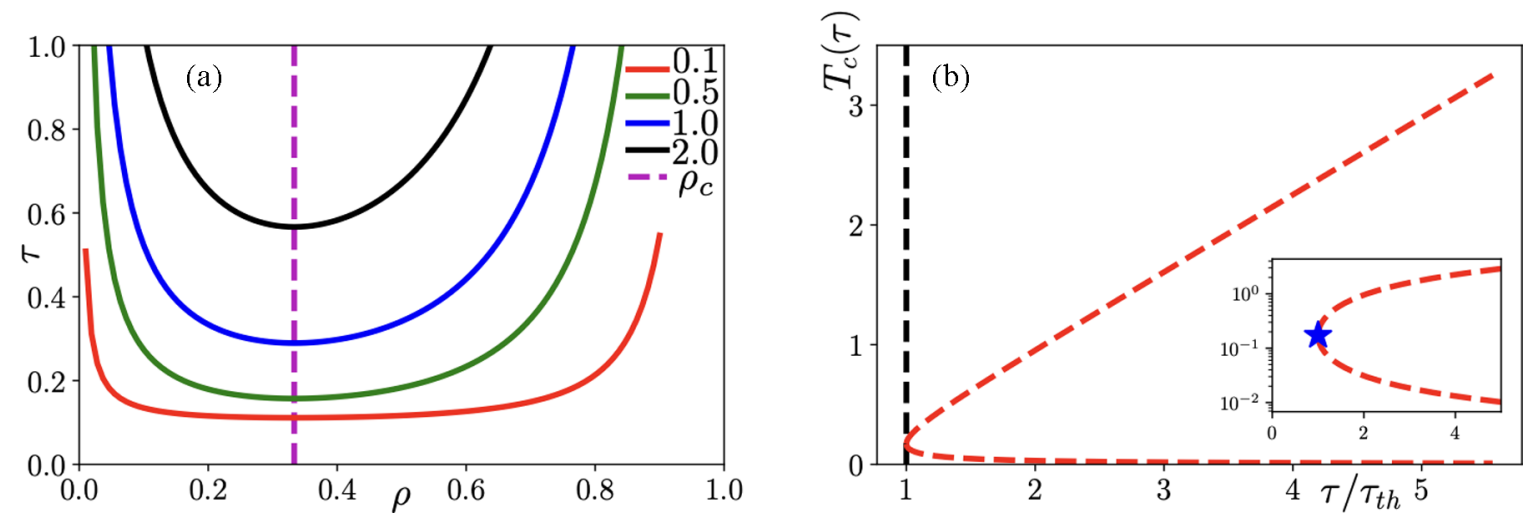

FIG. 3. Phase diagram for purely repulsive potentials (MIPS). (a) Phase diagram in the plane $\tau$ vs $\rho$ with $\tau$. Different lines refer to different noise strength $T$. The dashed magenta line indicates the critical density $\rho_{c}=1 / 3$. The solid curves are the spinodals. (b) Critical temperature as a function of the persistence time $\tau$ (dashed red line); the dashed black vertical line indicates the threshold value $\tau_{\text {th }}$. In the inset we show a zoom of the reentrant region; the star indicates $\tau_{\text {th }}$.

\section{B. Lennard-Jones potentials: Active gas-liquid transition}

Now we consider mechanical interactions due to pair potentials that are repulsive at small distances and attractive in a finite range. Focusing our attention on the case of LennardJones interactions, one has

$$
\phi_{\text {smooth }}(r)=\left(\frac{\sigma}{r}\right)^{12}-\left(\frac{\sigma}{r}\right)^{6}
$$

Again, for $\tau=0$ the system reduces to a Lennard-Jones fluid in equilibrium at temperature $T$. In equilibrium, one recovers the standard van der Waals theory that locates the critical point at $\rho_{c}=1 / 3, T_{c}=8 \alpha / 27$. Also in the case of ALJ fluids, for $\tau \neq 0$, the critical density remains the same while the critical temperature becomes a function of $\tau$, i.e., $T_{c}=T_{c}(\tau)$. Considering the solutions of $\partial_{\rho} P\left(\rho_{c}\right)=0$, we compute the critical temperature

$$
T_{c}(\tau)=\frac{4\left(\sqrt{(d-12)(d-6)\left\{4(d-12)(d-6)[d(d+16)-108]^{2} \tau^{2}+9(d-14)^{2}(d-8)^{2}\right\}}-2(d-12)(d-6)[d(d+16)-108] \tau\right)}{9(d-14)(d-12)(d-8)(d-6)} .
$$

Since ALJ fluids undergo gas-liquid coexistence in equilibrium, one has $T_{c}(0) \neq 0$.

The resulting phase diagram is shown in Fig. 4. The solid lines in the left panel represent the spinodal lines; different colors refer to different values of $T$. The dashed purple line is the critical density. In the right panel is shown the behavior of the critical temperature as a function of $\tau$, i.e., $T_{c}(\tau)$.

\section{Universality class of motility-induced phase separation}

For investigating the universality class of MIPS within the framework presented here, we have to expand the action $S_{\text {eff }}$ around the critical density $\rho_{c}$ and thus we write $\rho(x)=\rho_{c}+$ $\varphi(x)$, where $\varphi(x)$ represents a density fluctuation near the transition. In doing that, let us introduce the Gibbs free energy $G[\rho]=S_{\text {eff }}[\rho]$. Moreover, we have to introduce the chemical
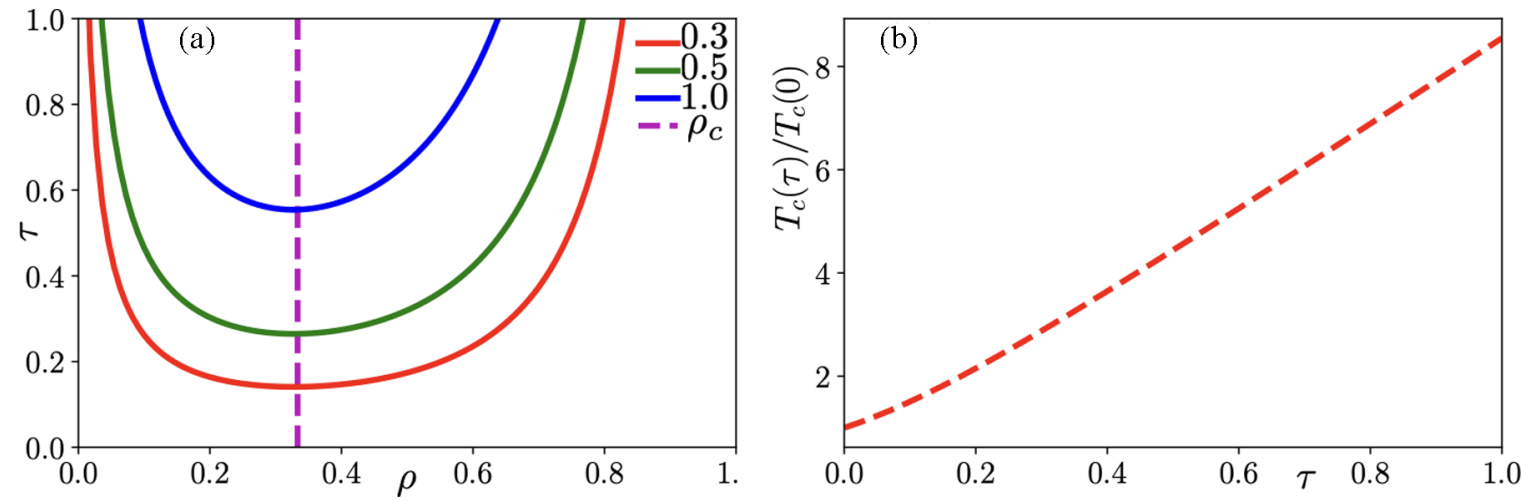

FIG. 4. Phase diagram of Lennard-Jones active fluids. (a) Phase diagram in the plane $\tau$ vs $\rho$. Different lines refer to different noise strength $T$. The dashed magenta line indicates the critical density $\rho_{c}=1 / 3$. The solid curves are the spinodals. (b) Critical temperature as a function of the persistence time $\tau$. 
potential $\mu$ that guarantees density fluctuations around the critical point, i.e., we have to consider the Legendre transform of $G[\rho] \rightarrow G[\rho]-\mu \rho$. In this way, expanding $G$ in powers of $\varphi(x)$, one has the cancellation of the linear term in $\varphi(x)$ that is balanced by the chemical potential term $\mu \varphi(x)$. Around the critical point for $b=0$, the expansion $G=\sum_{l} \frac{1}{l !} a_{l} \varphi^{l}$ contains only even powers of $\varphi$, and thus it takes the form $G[\varphi]=\int d \mathbf{x}\left\{\frac{c}{2}(\nabla \varphi)^{2}+\sum_{l=1}^{N_{l}} \frac{1}{2 l !} a_{2 l}[\varphi(\mathbf{x})]^{2 l}\right\}$. As a result, the effective theory reduces to a Landau-Ginzburg $\varphi^{4}$ theory that puts MIPS in the universality class of the Ising model.

However, if we consider elongated particles or density fluctuations that are not isotropic, the term $b \varphi^{3}$ might be different from zero, providing an additional source for the breaking of $\varphi \rightarrow-\varphi$ symmetry even close to the critical density. As a consequence, we might observe deviations from the Ising universality class, in the sense that the critical point might be replaced by a first-order transition. This prediction of the mean-field theory has strong consequences in several experimental situations, for instance, in the case of myxococcus xanthus where a MIPS-like phase separation has been observed [64]. In the next section, we will discuss predictions about the theory for $b \neq 0$.

\section{MIPS IN THE PRESENCE OF ANISOTROPIC INTERACTIONS}

When we perform a mean-field approximation on the partition function Eq. (42), we observe that, in the case of a central pair potential, the integral in front of the contribution $\rho^{3}$ in Eq. (48) vanishes identically for symmetry reasons. It is worth noting that, if the pair potential has not been taken isotropic, i.e., as in the case of elongated particles, the integral might assume a finite value. Using again Eq. (48) as a starting point, we consider homogeneous solutions of the saddle-point equations. We thus consider a phenomenological coarse-grained theory with $b$ as an external control parameter that tunes the degree of anisotropy of the two-body interaction. In this way, we can provide a qualitative estimate of the effect of particle asymmetries on spinodally decomposed active systems. We will start by focusing our attention on the case of a system undergoing MIPS and thus described by $\alpha(\beta, \tau)$ given by Eq. (50). Looking at the solution of $\partial_{\rho} P(\rho)=0$, we can compute the mean-field coexistence curves $\tau(\rho, T, b)$ that now depend also on the control parameter $b$. Considering $d=2$, we obtain the following expression for the coexistence curve:

$$
\tau(\rho, T, b)=\frac{\rho\left(2 \rho-\rho^{2}-1\right)-5 T^{2}}{15 T \rho(\rho-1)^{2}(b \rho-8)},
$$

that reduces to the previous results for $b=0$. The behavior of Eq. (57) is shown in Fig. 5, upper panel. As one can appreciate, the coexistence region for $b>0$ (blue curve) occupies a smaller area with respect the case of $b=0$. This effect can be rationalized looking at the free-energy Eq. (48). The term $b \rho^{3}$ increases the energy and thus the system tends to minimize the value of $\rho$. This term, in the coexistence region, renormalizes the value of the $\varphi^{3}$ term that is naturally presented considering the Landau expansion in that region. However, close to the critical point, where the $\varphi^{3}$ term of the Landau theory goes to zero, $b \varphi^{3}$ provides an extra contribution
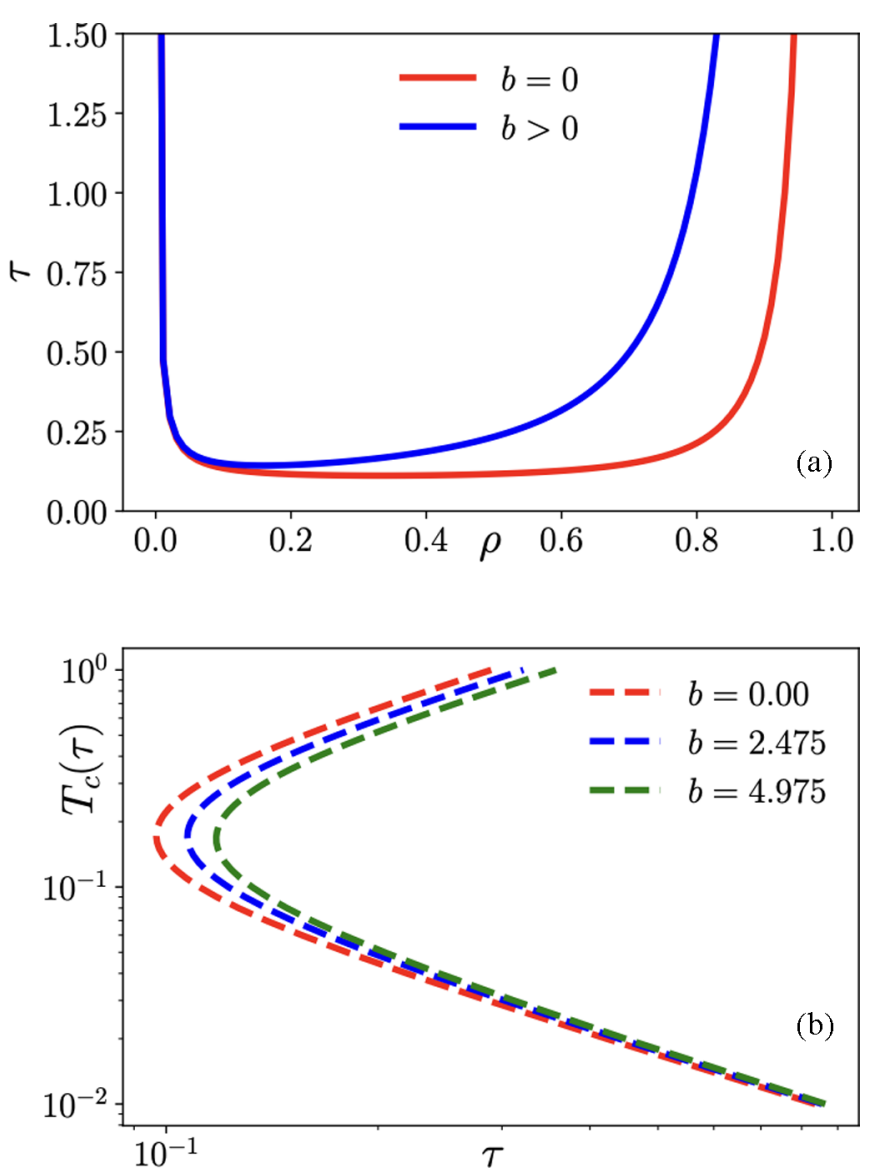

FIG. 5. (a) The coexistence curve Eq. (57) in the presence of asymmetric terms in the Landau-Ginzburg free energy. Phase diagram in the $\tau$ vs $\rho$ plane with $b=0$ (red curve) and $b=8$ (blue curve). (b) MIPS critical point $T_{c}$ as a function of $\tau$ as the asymmetry parameter $b$ increases.

that is in general different from zero. In the case of a $\varphi^{3}$ theory where one has an explicit breaking of $\varphi \rightarrow-\varphi$ symmetry, one of the two phases becomes energetically preferred. This is signaled by the presence of a metastable minimum in the corresponding Landau-Ginzburg free energy. The details are given in Appendix G 2. It is worth noting that, depending on the value assumed by $b$ with respect to $\alpha$, the $\varphi^{3}$ term might destroy the second-order transition that would be replaced by a first-order one.

Using Eq. (57), we can compute numerically the critical line $T_{c}(\tau, b)$, i.e., the location of the critical point as a function of the remaining control parameters. The result is shown in Fig. 5, lower panel, for $b=0.00,2.475,4.975$. Again, the critical curve remains reentrant; however, the asymmetric interaction tends to move the transition at higher $\tau$ values. This effect is made evident looking at the shift of the threshold value $\tau_{\text {th }}$ as a function of $b$, as it is shown in Fig. 6.

\section{DISCUSSION AND CONCLUSIONS}

In this paper, using the machinery of statistical field theory, we have developed a unified theoretical framework for studying peculiar nonequilibrium phenomena in scalar active matter, i.e., an active system described on large scales by 


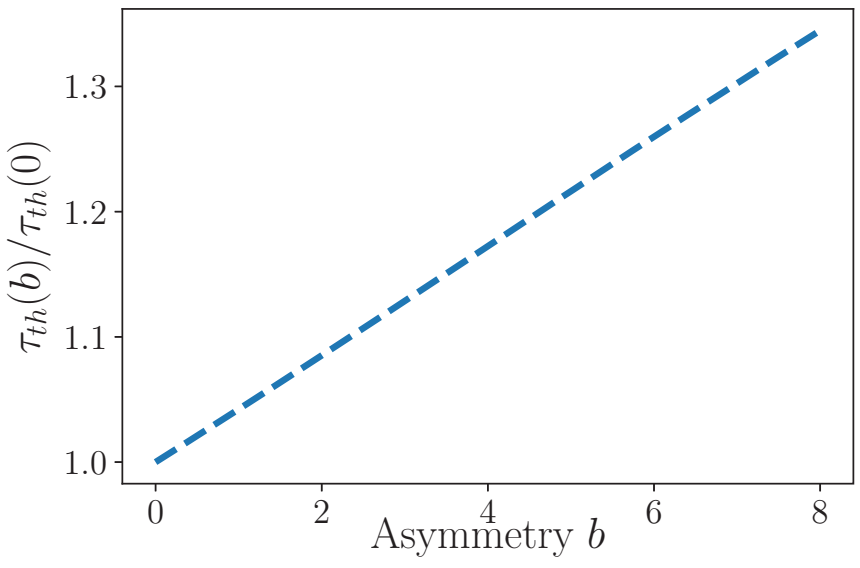

FIG. 6. Threshold value $\tau_{\text {th }}$, i.e., the minimum value of the persistence time for observing a spinodal decomposition, as a function of the asymmetry parameter $b$.

a scalar order parameter $\varphi$. We have derived an effective equilibrium action that contains nonlocal terms. The nonlocal terms can be tackled using a mean-field approximation that, in the presented framework, turns out is well defined, in the sense that we do not introduce uncontrolled approximation. The mean-field picture is obtained considering the saddle-point solution of the corresponding field theory. Finally, looking at homogeneous solutions and the stability of homogeneous configurations, we are able to compute the phase diagram of the system. We have focused our attention on three phenomena that concern the condensation of active particles: (i) accumulation of active particles at the boundaries of a container, (ii) motility-induced phase separation, and (iii) gasliquid phase transitions in active Lennard-Jones fluids.

In the picture presented here, the phenomenology of scalar active matter is captured by an equilibrium field theory based on UCN approximation. Nonequilibrium phase transitions with MIPS are due to nonlocal many-body contributions in the action. As a general result, looking at the active system at criticality, scalar active matter is described by the following Landau-Ginzburg free energy:

$$
f_{\mathrm{LG}}^{\text {active }}(\varphi)=\frac{a(T, \tau)}{2} \varphi^{2}+\frac{b(\tau)}{3} \varphi^{3}+\frac{c}{4} \varphi^{4}
$$

In the case of repulsive potential, the coefficient $a(T, \tau)$ is positive for $\tau=0$, i.e., when the system is in equilibrium. In the case of Lennard-Jones systems, $a(T, \tau)$ changes sign at $T_{c}$ in equilibrium $(\tau=0)$. For $\tau>0, a(T, \tau)$ vanishes along a critical line $T_{c}(\tau)$. The shape of the critical line depends on the microscopic details of the system. In the case of purely repulsive systems, $a(T, \tau)=0$ at the MIPS critical point that, at the mean-field level, turns out to be in the Ising universality class. According to this picture, in the case $b(\tau)=0$, MIPS results from the spontaneous symmetry breaking of $\varphi \rightarrow$ $-\varphi$ symmetry. We obtained that MIPS takes place above a threshold value of persistence time $\tau_{\text {th }}$. Moreover, MIPS is characterized by a reentrant phase diagram in the $T$ vs $\tau$ plane, i.e., above $\tau_{\text {th }}$. This means that the system evaporates into a gas state by decreasing the driving force.

On the other hand, when $b(\tau) \neq 0, f_{\mathrm{LG}}^{\text {active }}(\varphi)$ predicts also the presence of a $\varphi^{3}$ term that explicitly breaks the $\varphi \rightarrow-\varphi$ symmetry. We showed that the cubic term should be taken into account when active particles interact through an anisotropic potential, e.g., in the case of elongated swimmers. As a possible scenario, the MIPS critical point can be destroyed. In this case, it might be substituted by a first-order phase transition where a metastable MIPS state is nucleated at a higher $T$. This state will become eventually the stable one at smaller $T$. Looking at the properties of the system inside the coexistence region, the system is described by a van der Waals-like equation in both cases: MIPS and active LennardJones fluids. It is worth noting that the mean-field picture developed here predicts MIPS at low persistence times. This is basically due to the fact that we are considering a small $\tau$ expansion for computing det $\mathbf{M}$ in Eq. (21). This is surely in quantitative disagreement with numerical evidences. In particular, comparing the phase diagram shown in Fig. 3 with numerical simulations of active Brownian particles in Ref. [65], the mean-field theory developed here qualitatively reproduces the phase diagram in two dimensions. However, in numerical simulations, MIPS takes place at larger Péclet numbers, and thus larger $\tau$ values. However, the mean-field picture qualitatively reproduces the main physical mechanisms responsible for phase separation in active matter.

In the case of noninteracting active particles, we showed that the accumulation of particles away from the minimum of the trapping potential can be rationalized in terms of a spontaneous symmetry-breaking mechanism. In particular, performing a zero-temperature approximation, the density distribution $\rho(x) \sim \delta\left(x-x_{\alpha}\right)$ is concentrated at $x_{\alpha} \neq 0$ as soon as $\tau \neq 0$.

In conclusion, we have shown that effective equilibrium field theories are suitable for gaining insight into condensation phenomena typical of active systems.

\section{ACKNOWLEDGMENTS}

M.P. acknowledges funding from Regione Lazio, Grant No. 85-2017-15257 ("Progetti di Gruppi di Ricerca-Legge 13/2008-art. 4"). This work was also supported by the Joint Laboratory on "Advanced and Innovative Materials," ADINMAT, WIS-Sapienza (M.P.). We thank Umberto Marini Bettolo Marconi for his critical reading of the paper.

\section{APPENDIX A: $\boldsymbol{n}$-BODY INTERACTIONS IN EQUILIBRIUM STATISTICAL MECHANICS}

In this Appendix, we discuss the theoretical framework that we have employed for performing the coarse graining of the UCN Hamiltonian defined in Eq. (21). Let us start with the more general problem of $n$-body interactions in Hamiltonian systems, i.e., the Hamiltonian given in Eq. (2). We focus our attention on a system described by a scalar field, i.e., the density $\rho(r)$. As a standard starting point [57], we consider the identity

$$
1=\int d r \delta\left(r-r_{i}\right), \forall i
$$


and thus we can rewrite the interactions in Eq. (2) as follows:

$$
\begin{aligned}
\sum_{i=1}^{N} \phi_{1}\left(r_{i}\right) & =\sum_{i=1}^{N} \int d r \phi_{1}(r) \delta\left(r-r_{i}\right)=\int d r \phi_{1}(r) \sum_{i} \delta\left(r-r_{i}\right), \\
\sum_{i, j}^{1, N} \phi_{2}\left(r_{i}, r_{j}\right) & =\sum_{i, j} \int d r d r^{\prime} \phi_{2}\left(r, r^{\prime}\right) \delta\left(r-r_{i}\right) \delta\left(r-r_{j}\right)=\int d r d r^{\prime} \phi_{2}\left(r, r^{\prime}\right) \sum_{i, j} \delta\left(r-r_{i}\right) \delta\left(r-r_{j}\right), \\
\sum_{i, j, k} \phi_{3}\left(r_{i}, r_{j}, r_{k}\right) & =\sum_{i, j, k} \int d r d r^{\prime} d r^{\prime \prime} \phi_{3}\left(r, r^{\prime}, r^{\prime \prime}\right) \delta\left(r-r_{i}\right) \delta\left(r-r_{j}\right) \delta\left(r-r_{k}\right) \\
& =\int d r d r^{\prime} d r^{\prime \prime} \phi_{3}\left(r, r^{\prime}, r^{\prime \prime}\right) \sum_{i, j, k} \delta\left(r-r_{i}\right) \delta\left(r-r_{j}\right) \delta\left(r-r_{k}\right) .
\end{aligned}
$$

It is worth noting that $N=\sum_{i} \int d r \delta\left(r-r_{i}\right)=\int d r \sum_{i} \delta\left(r-r_{i}\right)$. The density field $\rho(r)$ is provided by the following relation:

$$
\rho(r) \equiv\left\langle\delta\left(r-r_{i}\right)\right\rangle=\frac{\int \prod_{i} d r_{i} e^{-\beta \mathcal{H}+\beta \mu N} \delta\left(r-r_{i}\right)}{Z} .
$$

In order to perform a coarse graining of the microscopic model, we introduce the field $\psi(r)$ through the identity

$$
1=\int \mathcal{D} \psi(r) \delta\left[\psi(r)-\sum_{i} \delta\left(r-r_{i}\right)\right],
$$

and we can thus rewrite the partition function in terms of the coarse-grained scalar field $\psi(r)$ as follows:

$$
\begin{aligned}
Z & =\int \prod_{i} \frac{d r_{i}}{\lambda^{N}} \int \mathcal{D} \psi(r) \delta\left[\psi(r)-\sum_{i} \delta\left(r-r_{i}\right)\right] e^{-S[\psi]-\int d r b(r) \psi(r),} \\
b(r) & \equiv \beta \phi_{1}(r)-\beta \mu, \\
S[\psi] & \equiv \frac{1}{2} \int d r d r^{\prime} \psi(r) \Delta^{-1}\left(r, r^{\prime}\right) \psi\left(r^{\prime}\right)+V[\psi], \\
\Delta^{-1}\left(r, r^{\prime}\right) & \equiv \beta \phi_{2}\left(r, r^{\prime}\right), \\
V[\psi] & \equiv \sum_{k \geqslant 3} \frac{1}{k !} \int d r_{1} \ldots d r_{k} \beta \phi_{k}\left(r_{1}, \ldots, r_{k}\right) \psi\left(r_{1}\right) \ldots \psi\left(r_{k}\right) .
\end{aligned}
$$

Now, using standard techniques in statistical field theory [27,40], we are going to employ the following representation for the delta distribution:

$$
\delta(x)=\int_{s_{0}-\mathbf{i} \infty}^{s_{0}+\mathbf{i} \infty} \frac{d s}{2 \pi \mathbf{i}} e^{s x} .
$$

We can thus represent the delta functional in $Z$ introducing an auxiliary field $\hat{\psi}(r)$ and writing

$$
\delta\left[\psi(r)-\sum_{i} \delta\left(r-r_{i}\right)\right]=\int \frac{\mathcal{D} \hat{\psi}(r)}{2 \pi \mathbf{i}} e^{\int d r \hat{\psi}(r)\left[\psi(r)-\sum_{i} \delta\left(r-r_{i}\right)\right]}
$$

Now the partition function reads

$$
\begin{aligned}
Z= & \int \mathcal{D} \psi(r) \mathcal{D} \hat{\psi}(r) e^{-G[\psi, \hat{\psi}]}, \\
-G \equiv & -S[\psi]+\int d r[\hat{\psi}(r)-b(r)] \psi(r) \\
& +N \ln \int \frac{d r}{\lambda} e^{-\hat{\psi}(r)}
\end{aligned}
$$

The field $\hat{\psi}(r)$ can be shifted in a way that $\hat{\psi}-b \rightarrow \hat{\psi}$ and thus the partition function becomes

$$
\begin{aligned}
Z= & \int \mathcal{D} \psi(r) \mathcal{D} \hat{\psi}(r) e^{-G[\psi, \hat{\psi}]}, \\
-G \equiv & -S[\psi]+\int d r \hat{\psi}(r) \psi(r) \\
& +N \ln \int \frac{d r}{\lambda} e^{-\hat{\psi}(r)-b(r)} .
\end{aligned}
$$


For making further progress, we introduce the following generating functional $W[\hat{\psi}]$ :

$$
e^{W[\hat{\psi}]} \equiv \mathcal{N}^{-1} \int \mathcal{D} \psi e^{-S[\psi]+\int d r \hat{\psi}(r) \psi(r)}
$$

where we have introduced the normalization $\mathcal{N} \equiv$ $\int \mathcal{D} \psi e^{-S[\psi]}$. As one can appreciate, the auxiliary field $\hat{\psi}$ in Eq. (A10) plays the role of external source in a quantum field theory. It is worth noting that $W[\hat{\psi}]=O(N)$. We can now introduce $W[\hat{\psi}]$ in the expression of $Z$, obtaining

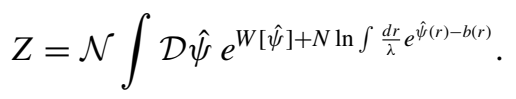

Finally, we define the functional $F[\hat{\psi}]$ through the relation

$$
F[\hat{\psi}]=W[\hat{\psi}]+N \ln \int \frac{d r}{\lambda} e^{-\hat{\psi}(r)+b(r)} .
$$

\section{APPENDIX B: FLUCTUATIONS}

In this section, we compute the fluctuations around the saddle-point configuration $\psi_{\mathrm{SP}}$. Let $\hat{\psi}_{\mathrm{SP}}(r)$ be the field configuration that makes the action $F[\hat{\psi}]$ stationary, and we can write

$$
\hat{\psi}(r)=\hat{\psi}_{\mathrm{SP}}(r)+\Delta \hat{\psi}(r) .
$$

With the field configuration $\hat{\psi}_{\mathrm{SP}}(r)$ the solution of the saddlepoint equation

$$
\left.\frac{\delta F}{\delta \hat{\psi}(r)}\right|_{\hat{\psi}=\hat{\psi}_{\mathrm{SP}}}=0 .
$$

We can now expand the effective action $F[\hat{\psi}]$ up to the second order, obtaining

$$
F[\hat{\psi}]=F\left[\hat{\psi}_{\mathrm{SP}}\right]+\frac{1}{2} \int d r d r^{\prime} \Delta \hat{\psi}(r) \mathcal{G}\left(r, r^{\prime}\right) \Delta \hat{\psi}\left(r^{\prime}\right)
$$

where the quadratic part is defined as follows:

$$
\left.\mathcal{G}(r, s) \equiv \frac{\delta^{2} F[\hat{\psi}]}{\delta \hat{\psi}(r) \delta \hat{\psi}(s)}\right|_{\mathrm{SP}} .
$$

It is worth noting that the computation of the stationary configurations brings us to the following self-consistency equation:

$$
\frac{\delta}{\delta \hat{\psi}_{\mathrm{SP}}} W\left[\hat{\psi}_{\mathrm{SP}}\right]=N \frac{e^{-\hat{\psi}_{\mathrm{SP}}-b(r)}}{\int d r e^{-\hat{\psi}_{\mathrm{SP}}-b(r)}} .
$$

Now we compute the Gaussian fluctuations around the stationary solution. In order to perform the computation we notice that

$$
\frac{\delta F}{\delta \hat{\psi}(r)}=\frac{\delta W}{\delta \hat{\psi}(r)}+N \frac{1}{z} \frac{\delta z}{\delta \hat{\psi}(r)}
$$

where we have defined

$$
z \equiv \int \frac{d r}{\lambda} e^{-\hat{\psi}(r)+b(r)} .
$$

The functional derivatives of $z$ with respect to the field $\hat{\psi}(r)$ give the following relations:

$$
\begin{aligned}
-\frac{\delta z}{\delta \hat{\psi}(r)} & =e^{-\hat{\psi}(r)+b(r)}, \\
\frac{\delta^{2} z}{\delta \hat{\psi}(r) \delta \hat{\psi}(s)} & =e^{-\hat{\psi}(r)+b(r)} \delta(r-s) .
\end{aligned}
$$

Now we define $\rho(r)$ and $G(r, s)$ as follows:

$$
\begin{aligned}
&\left.\frac{\delta W}{\delta \hat{\psi}(r)}\right|_{\mathrm{SP}} \equiv \rho(r), \\
&\left.\frac{\delta^{2} W}{\delta \hat{\psi}(r) \delta \hat{\psi}(s)}\right|_{\mathrm{SP}} \equiv G(r, s),
\end{aligned}
$$

and thus the quadratic part in Eq. (B4) can be rewritten as

$$
\frac{\delta F^{2}[\hat{\psi}]}{\delta \hat{\psi}(r) \delta \hat{\psi}(s)}=G(r, s)-\rho(r) \delta(r-s)+\frac{1}{N} \rho(r) \rho(s) .
$$

After introducing $G(r, s)$ and $\rho(r)$, we can finally write the partition function in terms of two contributions: the first one is due to the saddle-point configuration $\hat{\psi}_{\text {SP }}$ of the field; the second one is due to fluctuation around it. We can thus write

$$
\begin{aligned}
Z & =Z_{\mathrm{SP}} Z_{\text {fluct }}, \\
Z_{\mathrm{SP}} & \equiv \mathcal{N} e^{W\left[\hat{\psi}_{\mathrm{SP}}\right]+N \ln z\left[\hat{\psi}_{\mathrm{SP}}\right]}, \\
Z_{\text {fluct }} & \equiv \int \mathcal{D} \hat{\psi} e^{\frac{1}{2} \int d r d s \hat{\psi}(r)\left[G(r, s)-\rho(r) \delta(r-s)+\frac{1}{N} \rho(r) \rho(s)\right] \hat{\psi}(s)}
\end{aligned}
$$

where we have changed notation $\Delta \psi \rightarrow \hat{\psi}$. Through the propagator $G^{-1}(r, s)$ we can perform the following transformation:

$$
\hat{\psi}(r)=\mathbf{i} \int d s G^{-1}(r, s) \varphi(s) .
$$

Employing such a change of variable one obtains

$$
\begin{aligned}
\int & d r d s \hat{\psi}(r) G(r, s) \hat{\psi}(s) \\
= & -\int d r d s \int d s^{\prime} G^{-1}\left(r, s^{\prime}\right) \varphi\left(s^{\prime}\right) G(r, s) \\
& \times \int d s^{\prime \prime} G^{-1}\left(s, s^{\prime \prime}\right) \varphi\left(s^{\prime \prime}\right) \\
= & \int d s^{\prime} d s^{\prime \prime} \varphi\left(s^{\prime}\right) G^{-1}\left(s^{\prime}, s^{\prime \prime}\right) \varphi\left(s^{\prime \prime}\right) .
\end{aligned}
$$

The second term in Eq. (B10) becomes

$$
\begin{aligned}
& \int d r \hat{\psi}(r) \rho(r) \hat{\psi}(r) \\
&=\int d r d s d s^{\prime} \varphi(s) G^{-1}(r, s) G^{-1}\left(r, s^{\prime}\right) \rho(r) \varphi\left(s^{\prime}\right),
\end{aligned}
$$

and the third term reads

$$
\begin{aligned}
\int d r d r^{\prime} \hat{\psi}(r) \rho(r) \rho\left(r^{\prime}\right) \hat{\psi}\left(r^{\prime}\right) \\
=\int d s d s^{\prime} \varphi(s)\left[\int d r G^{-1}(r, s) \rho(r)\right] \\
\times\left[\int d r G^{-1}\left(r, s^{\prime}\right) \rho(r)\right] \varphi\left(s^{\prime}\right) .
\end{aligned}
$$


It is worth noting that the last term in Eq. (B10) gives the subleading contribution since it is multiplied by $1 / N$. Finally, we have to take into account the Jacobian determinant of the transformation $\hat{\psi} \rightarrow \varphi$. Putting all together we arrive at the following expression for the partition function:

$$
\begin{aligned}
& Z=\frac{\mathcal{N}}{\operatorname{det} G} e^{F\left[\hat{\psi}_{\mathrm{SP}}\right]} \int \mathcal{D} \varphi e^{-\frac{1}{2} \int d r d r^{\prime} \varphi \mathcal{G} \varphi}, \\
& \mathcal{G} \equiv G^{-1}\left(r, r^{\prime}\right)-\int d s G^{-1}(s, r) \rho(s) G^{-1}\left(s, r^{\prime}\right)+O(1 / N) \\
& F\left[\hat{\psi}_{\mathrm{SP}}\right] \equiv W\left[\hat{\psi}_{\mathrm{SP}}\right]+N \ln z\left[\hat{\psi}_{\mathrm{SP}}\right] \\
& \hat{\psi}_{\mathrm{SP}}:\left.\frac{\delta F}{\delta \hat{\psi}}\right|_{\mathrm{SP}}=0 \\
& \rho=\frac{\delta}{\delta \hat{\psi}_{\mathrm{SP}}} W\left[\hat{\psi}_{\mathrm{SP}}\right], \\
& G \equiv \frac{\delta^{2}}{\delta \hat{\psi}_{\mathrm{SP}}^{2}} W\left[\hat{\psi}_{\mathrm{SP}}\right], \\
& e^{W[\hat{\psi}]}=\mathcal{N}-1 \\
& \mathcal{N} \equiv \int \mathcal{D} \psi e^{-S[\psi]+\int d x \hat{\psi} \psi} \\
& S[\psi] \equiv \beta H[\psi]-\left.\psi \frac{\mathrm{B} 16)}{\delta \psi} \beta H\right|_{\psi=0} \cdot
\end{aligned}
$$

In writing Eqs. (B16) we have neglected $O(1 / N)$ terms:

$$
\frac{1}{N}\left[\int d s \rho(s) G^{-1}(s, r)\right]\left[\int d s \rho(s) G^{-1}\left(s, r^{\prime}\right)\right] \propto \frac{1}{N} .
$$

\section{APPENDIX C: GAS IN THERMAL EQUILIBRIUM IN EXTERNAL POTENTIALS}

Now we consider a gas composed by noninteracting particles in one spatial dimension that are embedded into an external potential $A(x)$. The external potential is a smooth and continuous function of class $\mathcal{C}^{\infty}$. The Hamiltonian $H$ reads

$$
H\left[x_{i}\right]=\sum_{i=1}^{N} A\left(x_{i}\right) .
$$

The partition function is

$$
Z_{\beta}=\int \prod_{i} d x_{i} e^{-\beta H\left[x_{i}\right]},
$$

in terms of the number density field $\psi(x)$ and the auxiliary field $\hat{\psi}(x)$, and we can write

$$
\begin{aligned}
Z_{\beta} & =\int \mathcal{D} \psi(x) \mathcal{D} \hat{\psi}(x) e^{-G[\psi, \hat{\psi}]}, \\
G[\psi, \hat{\psi}] & \equiv \hat{\psi} \cdot \psi+\beta \psi \cdot A-N \ln z[\hat{\psi}], \\
z[\hat{\psi}] & \equiv \int d x e^{\hat{\psi}(x)}, \\
f \cdot g & \equiv \int d x f(x) g(x) .
\end{aligned}
$$

Indicating with $n(x) \equiv \psi(x)_{\mathrm{SP}}$ and $\hat{n}(x) \equiv \hat{\psi}(x)_{\mathrm{SP}}$ the filed configurations that satisfy the self-consistency equations, we have

$$
n(x)-N \frac{e^{\hat{n}(x)}}{z}=0,
$$

and we can thus write

$$
\hat{n}(x)=\ln z[\hat{n}]+\ln n(x)-\ln N .
$$

Since $\int d x n(x)=N$ we can finally write

$$
G[n]=\int d x n(x)[\beta A(x)+\ln n(x)]
$$

and the corresponding self-consistency equation is

$$
n(x)=c e^{-\beta A(x)}
$$

with $c=$ const $>0$. At small temperature $\beta \rightarrow \infty$ the density profile is dominated by the minima of $A(x)$ and thus $n(x) \sim$ $\sum_{\alpha} \delta\left(x-x_{0}^{\alpha}\right)$ with $x_{0}^{\alpha}$ given by

$$
\left.\frac{d A}{d x}\right|_{x=x_{0}^{\alpha}}=0,\left.\quad \frac{d^{2} A(x)}{d x^{2}}\right|_{x=x_{0}^{\alpha}} \geqslant 0 .
$$

\section{Soft confining potentials}

As a case study we are going to consider a gas in equilibrium confined through a potential

$$
A(x)=\frac{x^{2 \alpha}}{2 \alpha}
$$

where the corresponding density profile is

$$
n(x)=c e^{-\beta \frac{x^{2 \alpha}}{2 \alpha}} .
$$

At high temperatures $\beta \rightarrow 0$ and then $n(x)=c$. The constant is fixed by the normalization; considering the particles free to move in a segment of length $L$ centered around the origin, one has

$$
\int_{-L / 2}^{L / 2} d x n(x)=c L=1
$$

and thus $c=\frac{1}{L}$. At zero temperature $n(x) \sim \delta\left(x-x_{0}\right)$ with $x_{0}$ given by

$$
\left.\frac{d A}{d x}\right|_{x=x_{0}}=x_{0}^{2 \alpha-1}=0,
$$

that has $x_{0}=0$ as the unique and stable solution $\forall \alpha>0$.

\section{APPENDIX D: MEAN-FIELD THEORY OF TWO-BODY INTERACTIONS}

Here we start with a specific problem that is the computation of the partition function $Z_{\beta}$ in the case of a particle system interacting through a pairwise potential. The Hamiltonian reads

$$
H(\mathbf{p}, \mathbf{r})=\sum_{i=1}^{N} \frac{\mathbf{p}_{i}^{2}}{2 m}+\sum_{i<j} \phi\left(\mathbf{r}_{i}, \mathbf{r}_{j}\right)
$$


and the partition function $Z_{\beta}$ is

$$
\begin{aligned}
Z_{\beta} & =\frac{1}{h^{3 N} N !} \int \prod_{i} d \mathbf{p}_{i} d \mathbf{r}_{i} e^{-\beta H(\mathbf{p}, \mathbf{r})} \\
& =\left(\frac{2 \pi m}{\beta}\right)^{\frac{3 N}{2}} \frac{1}{h^{3 N} N !} X_{\beta}
\end{aligned}
$$

where we have defined the configurational integral:

$$
X_{\beta} \equiv \int \prod_{i} d \mathbf{r}_{i} e^{-\beta \sum_{i<j} \phi\left(\mathbf{r}_{i}, \mathbf{r}_{j}\right)} .
$$

In order to perform a coarse graining of the microscopic dynamics, we introduce the density field $\rho(\mathbf{r})$ :

$$
\psi(\mathbf{r})=\sum_{i=1}^{N} \delta\left(\mathbf{r}-\mathbf{r}_{i}\right)
$$

and we can then rewrite the two-body interaction $\Phi \equiv$ $\sum_{i<j} \phi\left(\mathbf{r}_{i}, \mathbf{r}_{j}\right)$ in the following way:

$$
\begin{aligned}
\Phi & \equiv \frac{1}{2} \sum_{i, j} \phi\left(\mathbf{r}_{i}, \mathbf{r}_{j}\right) \\
& =\frac{1}{2} \int d \mathbf{r} d \mathbf{r}^{\prime} \psi(\mathbf{r}) \phi\left(\mathbf{r}, \mathbf{r}^{\prime}\right) \psi\left(\mathbf{r}^{\prime}\right)-N \phi(0) .
\end{aligned}
$$

The density field can be forced into the partition function using a delta functional

$$
\int \mathcal{D} \psi(\mathbf{r}) \delta\left[\psi(\mathbf{r})-\sum_{i} \delta\left(\mathbf{r}-\mathbf{r}_{i}\right)\right]=1
$$

that brings us to the following expression for the configurational integral:

$$
\begin{aligned}
X_{\beta}= & \int \prod_{i} d \mathbf{r}_{i} \mathcal{D} \psi(\mathbf{r}) \delta\left[\psi(\mathbf{r})-\sum_{i} \delta\left(\mathbf{r}-\mathbf{r}_{i}\right)\right] \\
& \times \exp \left(-\frac{\beta}{2} \int d \mathbf{r} d \mathbf{r}^{\prime} \psi(\mathbf{r}) \phi\left(\mathbf{r}, \mathbf{r}^{\prime}\right) \psi\left(\mathbf{r}^{\prime}\right)\right)
\end{aligned}
$$

The delta functional can be expressed using an auxiliary field $\hat{\psi}(\mathbf{r})$ :

$$
\begin{aligned}
& \delta\left[\psi(\mathbf{r})-\sum_{i} \delta\left(\mathbf{r}-\mathbf{r}_{i}\right)\right] \\
& \quad=\int \mathcal{D} \hat{\psi}(\mathbf{r}) e^{-\int d \mathbf{r} \psi(\mathbf{r}) \hat{\psi}(\mathbf{r})+\int d \mathbf{r} \hat{\psi}(\mathbf{r}) \sum_{i} \delta\left(\mathbf{r}-\mathbf{r}_{i}\right)},
\end{aligned}
$$

and the configurational integral becomes

$$
\begin{aligned}
X_{\beta}= & \int \prod_{i} d \mathbf{r}_{i} \mathcal{D} \psi(\mathbf{r}) \mathcal{D} \hat{\psi}(\mathbf{r}) \exp \left(-\int d \mathbf{r} \psi(\mathbf{r}) \hat{\psi}(\mathbf{r})\right. \\
& +\int d \mathbf{r} \hat{\psi}(\mathbf{r}) \sum_{i} \delta\left(\mathbf{r}-\mathbf{r}_{i}\right) \\
& \left.-\frac{\beta}{2} \int d \mathbf{r} d \mathbf{r}^{\prime} \psi(\mathbf{r}) \phi\left(\mathbf{r}, \mathbf{r}^{\prime}\right) \psi\left(\mathbf{r}^{\prime}\right)\right) .
\end{aligned}
$$

Let us introduce the one-body partition function $z[\hat{\psi}]$ :

$$
z[\hat{\psi}] \equiv\left(\int d \mathbf{r} e^{\hat{\psi}(\mathbf{r})}\right)^{N}
$$

and then we can write

$$
\begin{aligned}
X_{\beta}= & \int \mathcal{D} \psi(\mathbf{r}) \mathcal{D} \hat{\psi}(\mathbf{r}) e^{-G[\psi, \hat{\psi}]}, \\
G[\psi, \hat{\psi}] \equiv & \int d \mathbf{r} \psi(\mathbf{r}) \hat{\psi}(\mathbf{r})-\ln z[\hat{\psi}] \\
& +\frac{\beta}{2} \int d \mathbf{r} d \mathbf{r}^{\prime} \psi(\mathbf{r}) \phi\left(\mathbf{r}, \mathbf{r}^{\prime}\right) \psi\left(\mathbf{r}^{\prime}\right) .
\end{aligned}
$$

In the thermodynamic limit we assume that fluctuations are negligible. In this situation, the integral is dominated by the extreme values of $G$. Performing a saddle-point approximation, one has

$$
\begin{aligned}
& \left.\frac{\delta G}{\delta \hat{\psi}(\mathbf{r})}\right|_{\hat{\psi}=\hat{\rho}, \psi=\rho}=\rho(\mathbf{r})-\frac{N e^{\hat{\rho}(\mathbf{r})}}{\int d \mathbf{r} e^{\hat{\rho}(\mathbf{r})}}=0, \\
& \left.\frac{\delta G}{\delta \psi(\mathbf{r})}\right|_{\hat{\psi}=\hat{\rho}, \psi=\rho}=\hat{\rho}(\mathbf{r})+\beta \int d \mathbf{r}^{\prime} \phi\left(\mathbf{r}, \mathbf{r}^{\prime}\right) \rho\left(\mathbf{r}^{\prime}\right)=0 .
\end{aligned}
$$

Using the first equation, we can write

$$
\ln \rho(\mathbf{r})+\ln z-\ln N-\hat{\rho}(\mathbf{r})=0,
$$

and thus

$$
\hat{\rho}(\mathbf{r})=\ln \rho(\mathbf{r})+\ln z-\ln N .
$$

We can rewrite the free energy at the saddle point as a functional of the density field $\rho(\mathbf{r})$. Now we plug Eq. (D14) into Eq. (D11) for obtaining

$$
\begin{aligned}
G[\rho]= & \int d \mathbf{r} \rho(\mathbf{r})[\ln \rho(\mathbf{r})+\ln z-\ln N]-N \ln z \\
& +\frac{\beta}{2} \int d \mathbf{r} d \mathbf{r}^{\prime} \rho(\mathbf{r}) \phi\left(\mathbf{r}, \mathbf{r}^{\prime}\right) \rho\left(\mathbf{r}^{\prime}\right)
\end{aligned}
$$

and finally

$$
\begin{aligned}
G[\rho]= & \frac{\beta}{2} \int d \mathbf{r} d \mathbf{r}^{\prime} \rho(\mathbf{r}) \phi\left(\mathbf{r}, \mathbf{r}^{\prime}\right) \rho\left(\mathbf{r}^{\prime}\right) \\
& +\int d \mathbf{r} \rho(\mathbf{r}) \ln \rho(\mathbf{r})-N \ln N
\end{aligned}
$$

where we have used the constraint $\int d \mathbf{r} \rho(\mathbf{r})=N$.

In order to study the stability of a solution $(\rho(\mathbf{r}), \hat{\rho}(\mathbf{r}))$, one has to compute the Hessian matrix $\mathbf{H}$ with components $H_{\alpha, \beta}=\frac{\delta^{2} G}{\delta \rho_{\alpha}(\mathbf{r}) \delta \rho_{\beta}\left(\mathbf{r}^{\prime}\right)}$, where the greek indices take the values $\alpha=1,2$ with $\rho_{1} \equiv \rho$ and $\rho_{2} \equiv \hat{\rho}$. The stability condition is

$$
\left.\operatorname{det} \frac{\delta^{2} G}{\delta \rho_{\alpha}(\mathbf{r}) \delta \rho_{\beta}\left(\mathbf{r}^{\prime}\right)}\right|_{(\rho(\mathbf{r}), \hat{\rho}(\mathbf{r}))} \geqslant 0 .
$$

In the case of a two-body potential one has to consider the eigenvalues of the matrix:

$$
M(\mathbf{x}, \mathbf{y})=\beta \phi(\mathbf{x}, \mathbf{y})+\frac{\delta(\mathbf{x}-\mathbf{y})}{\rho(\mathbf{x})} .
$$


The two-body potential $\phi(\mathbf{x}, \mathbf{y})$ is translationally invariant; we can thus write $\phi(\mathbf{x}, \mathbf{y})=\phi(\mathbf{x}-\mathbf{y})$ and the matrix becomes

$$
M(\mathbf{x}, \mathbf{y})=M(\mathbf{x}-\mathbf{y})=\beta \phi(\mathbf{x}-\mathbf{y})+\frac{\delta(\mathbf{x}-\mathbf{y})}{\rho(\mathbf{x})} .
$$

Since the continuous matrix $M(\mathbf{x}-\mathbf{y})$ is translationally invariant, it is diagonal in Fourier space. Now we study the stability of a homogeneous density profile $\bar{\rho}$. Using the expressions

$$
\begin{aligned}
& \phi(\mathbf{x}-\mathbf{y})=\sum_{\mathbf{k}} e^{i \mathbf{k} \cdot(\mathbf{x}-\mathbf{y})} \hat{\phi}_{\mathbf{k}}, \\
& \delta(\mathbf{x}-\mathbf{y})=\sum_{\mathbf{k}} e^{i \mathbf{k} \cdot(\mathbf{x}-\mathbf{y})},
\end{aligned}
$$

the sums run over wave vectors $\mathbf{k}=\frac{2 \pi}{L} \mathbf{n}$. We obtain that homogeneous configurations are stable if

$$
\beta \hat{\phi}_{\mathbf{k}}+\frac{1}{\bar{\rho}} \geqslant 0 .
$$

Since we are interested in the large-scale behavior of the system, we look at $\mathbf{k} \rightarrow 0$ and thus

$$
\hat{\phi}_{0}=\Omega(d) \int d x x^{d-1} \phi(x),
$$

where $\Omega(d)$ results from the integration of the solid angle in $d$ spatial dimensions. As we will see in the next section, for a van der Waals gas the integral is negative and thus there is a critical density above which the homogeneous solution is not stable anymore.

\section{Stability of homogeneous density profiles}

Differently from the van der Waals theory, here we did not consider explicitly excluded volume effects. As a consequence, we cannot observe a spinodal decomposition between a liquid and a gas phase. However, we can still study the stability of homogeneous density profiles. For homogeneous solutions $\rho(\mathbf{r})=$ const $=\rho$, assuming translationally invariant interactions, we can write

$$
\begin{aligned}
G[\rho] & =V g(\rho), \\
g(\rho) & \equiv \frac{\beta \alpha}{2} \rho^{2}+\rho \ln \rho, \\
\alpha & \equiv \Omega(d) \int d r r^{d-1} \phi(r) .
\end{aligned}
$$

The pressure $P(\rho)$ can be computed as

$$
P(\rho)=\rho \frac{\partial g}{\partial \rho}-g(\rho)
$$

and thus we have

$$
\begin{aligned}
P(\rho) & =\rho(\beta \alpha \rho+1+\ln \rho)-\frac{\beta \alpha}{2} \rho^{2}-\rho \ln \rho \\
& =\rho\left(\frac{\beta \alpha}{2} \rho+1\right) .
\end{aligned}
$$

If we require the thermodynamical stability of the solution $\rho$, we have to compute the second derivative of $g(\rho)$ :

$$
\frac{\partial^{2} g}{\partial \rho^{2}}=\beta \alpha+\frac{1}{\rho}
$$

For purely repulsive potentials, $\alpha>0$ and thus $\frac{\partial^{2} g}{\partial \rho^{2}} \geqslant 0, \forall \rho \in$ $[0,1]$, i.e., homogeneous density profiles are always stable in equilibrium systems when attracting forces between particles are negligible.

Considering an equilibrium system where particles interact through a potential that is attractive on short distances, the integral in the third of Eqs. (D23) turns out to assume negative values, i.e., $\alpha<0$. Let us write $\alpha=-|\alpha|$; now the stability of homogeneous profiles is related to

$$
\frac{\partial^{2} g}{\partial \rho^{2}}=-\beta|\alpha|+\frac{1}{\rho}
$$

that changes sign at $\rho=\rho_{c}=\frac{1}{\beta|\alpha|}$. The location of the critical point can be computed considering the equations

$$
\frac{\partial P}{\partial \rho}=\frac{\partial^{2} P}{\partial \rho^{2}}=0
$$

that are

$$
1-\beta|\alpha| \rho=0, \quad-\alpha \beta=0
$$

The system of equations (D29) does not have solutions and thus, in the model we have considered, we do not have any critical point.

\section{Including excluded volume effects in the mean-field theory}

For obtaining the van der Waals theory it proves convenient to study the system discretized on a lattice. We follow a standard procedure that can be found in Refs. [56,57,63]. We illustrate the method considering an equilibrium system composed by $N$ particles in a volume $V$ in $d$ spatial dimensions. We perform a coarse graining dividing the system into a lattice that defines a set of occupation numbers $N_{i}$ that must satisfy the constraint $\sum_{i} N_{i}=N$. Each cell occupies a volume $\Delta$ and thus $V=N \Delta$, and each particle occupies a volume $\delta$. We consider the potential $\phi\left(\mathbf{r}_{i}, \mathbf{r}_{j}\right)$ composed by two parts:

$$
\phi\left(\mathbf{r}_{i}, \mathbf{r}_{j}\right)=\phi_{\mathrm{HS}}^{\delta}+\phi_{\text {smooth }}\left(\mathbf{r}_{i}, \mathbf{r}_{j}\right)
$$

where $\phi_{\mathrm{HS}}^{\delta}$ indicates a hard-core potential, i.e., each particle is a hard sphere that occupies a volume $\delta$. The second part $\phi_{\text {smooth }}(r)$ is a smooth function of $r$. The hard-core potential causes a contraction of the phase space $\omega\left(N_{i}\right)$ that now is $\omega\left(N_{i}\right)=\left(\Delta-N_{i} \delta\right)^{N_{i}}$. As we have done before when we have defined the local-density field $\psi(\mathbf{r})$, through the occupation numbers $N_{i}$ the energy can be written as

$$
\Phi=\frac{1}{2} \sum_{i, j} \phi_{i, j} N_{i} N_{j}
$$


with $\phi_{i, j} \equiv \phi_{\text {smooth }}\left(\mathbf{r}_{i}, \mathbf{r}_{j}\right)$. The configurational integral $X_{N}$ becomes

$$
\begin{aligned}
X_{\beta}= & \sum_{\left\{N_{i}\right\} \mid \sum_{i} N_{i}=N} e^{-G\left[N_{i}\right]}, \\
-G\left[N_{i}\right] \equiv & \sum_{i}\left[N_{i} \ln \left(\Delta-N_{i} \delta\right)-N_{i} \ln N_{i}+N_{i}\right] \\
& +\frac{\beta}{2} \sum_{i j} \phi_{i, j} N_{i} N_{j} .
\end{aligned}
$$

At the saddle point one has to find the solution of the set of equations

$$
\ln \left(\frac{\Delta-N_{i} \delta}{N_{i}}\right)-\left(\frac{N_{i} \delta}{\Delta-N_{i} \delta}\right)+\beta \sum_{i, j} \phi_{i, j} N_{j}=\mu
$$

where the Lagrangian multiplier $\mu$ guarantees $\sum_{i} N_{i}=N$. The uniform solution of the saddle-point equations has the form $N_{i}=\rho \Delta, \forall i$ with $\rho=N / V$. Setting $\delta=1$, the free energy becomes

$$
\begin{aligned}
-G[\rho] & =-V g(\rho), \\
-g(\rho) & \equiv \rho \ln \left(\frac{1-\rho}{\rho}\right)+\rho+\frac{\beta \alpha}{2} \rho^{2}, \\
\alpha & \equiv \sum_{i, j} \phi_{i, j} \Delta .
\end{aligned}
$$

Focusing our attention on the repulsive potential, we can write $\alpha=-|\alpha|$ and compute the van der Waals equation of state:

$$
\frac{\beta P(\rho)}{\rho}=\frac{1}{\rho-1}-\frac{\beta \alpha \rho}{2} .
$$

The critical point is determined through Eq. (D27) that brings us to $\beta_{c}=\frac{27}{4 \alpha}$ and $\rho_{c}=\frac{1}{3}$.

In order to connect Eq. (D34) with Eq. (D36) we perform the continuous limit $\Delta \rightarrow 0$ that brings us to

$$
\begin{aligned}
-G[\rho]= & \int d \mathbf{r} \rho(\mathbf{r})\left[\ln \frac{1-\rho(\mathbf{r})}{\rho(\mathbf{r})}+1\right] \\
& +\frac{\beta}{2} \int d \mathbf{r} d \mathbf{r}^{\prime} \phi_{\text {smooth }}\left(\mathbf{r}, \mathbf{r}^{\prime}\right) \rho(\mathbf{r}) \rho\left(\mathbf{r}^{\prime}\right) .
\end{aligned}
$$

As one can appreciate, Eq. (D36) has the form of a density functional theory. For obtaining the kinetic term $\nabla \rho$, it is convenient to go back to the discretized action and look at the interaction term

$$
\sum_{i, j} \phi_{i j} N_{i} N_{j}
$$

and using the identity

$$
N_{i} N_{j}=\frac{1}{2}\left(N_{i}^{2}+N_{j}^{2}\right)-\frac{1}{2}\left(N_{i}-N_{j}\right)^{2}
$$

the interaction term can be written as

$$
\sum_{i \neq j} \phi_{i j} N_{i} N_{j}=\sum_{i \neq j} \phi_{i j} N_{i}^{2}-\frac{1}{2} \sum_{i \neq j} \phi_{i j}\left(N_{i}-N_{j}\right)^{2} .
$$

Let $\rho_{i}$ be the density in the box $i: \rho_{i}=\frac{N_{i}}{\Delta}$. We can thus rewrite

$$
\begin{aligned}
& \sum_{i \neq j} \phi_{i j} N_{i}^{2}-\frac{1}{2} \sum_{i \neq j} \phi_{i j}\left(N_{i}-N_{j}\right)^{2} \\
& =\sum_{i \neq j} \Delta \Delta \phi_{i j} \rho_{i}^{2}-\frac{1}{2} \sum_{i \neq j} \Delta \Delta \phi_{i j}\left(\rho_{i}-\rho_{j}\right)^{2} .
\end{aligned}
$$

In the continuum limit, i.e., $\Delta \rightarrow 0$, we get

$$
\begin{gathered}
\sum_{i \neq j} \Delta \Delta \phi_{i j} \rho_{i}^{2}-\frac{1}{2} \sum_{i \neq j} \Delta \Delta \phi_{i j}\left(\rho_{i}-\rho_{j}\right)^{2} \\
\longrightarrow \epsilon \int d \mathbf{r} \rho(\mathbf{r})^{2}+\frac{c}{2} \int d \mathbf{r}(\nabla \rho)^{2} .
\end{gathered}
$$

The first term in the last equation is nothing else than a mass term in a field theory.

\section{APPENDIX E: UCN AS AN EFFECTIVE EQUILIBRIUM THEORY}

In this Appendix we show the computation that brings us to Eq. (38). For the sake of simplicity let us change the notation from $\mathbf{r}_{i}$ to $\mathbf{x}_{i}$ for indicating a configuration of the system. Now we consider the following Hamiltonian:

$$
\begin{aligned}
H_{\mathrm{UCN}}\left[\mathbf{x}_{i}\right] & =H_{0}\left[\mathbf{x}_{i}\right]+H_{1}\left[\mathbf{x}_{i}\right]+H_{2}\left[\mathbf{x}_{i}\right], \\
H_{0}\left[\mathbf{x}_{i}\right] & =\frac{1}{2} \sum_{i, j} \phi\left(\mathbf{x}_{i}, \mathbf{x}_{j}\right), \\
H_{1}\left[\mathbf{x}_{i}\right] & \equiv \frac{\tau}{2} \sum_{i}\left(\nabla_{\mathbf{x}_{i}} H_{0}\right)^{2}, \\
H_{2}\left[\mathbf{x}_{i}\right] & \equiv-T \ln \operatorname{det} M, \\
M & \equiv \delta_{i j}+\tau \frac{\partial^{2} H_{0}}{\partial \mathbf{x}_{i} \partial \mathbf{x}_{j}} .
\end{aligned}
$$

The thermodynamics is given as usual:

$$
\begin{aligned}
F & =-\frac{1}{\beta} \ln Z_{\beta}, \\
Z_{\beta} & =\int \prod_{i} d \mathbf{x}_{i} e^{-\beta H_{\mathrm{UCN}}\left[\mathbf{x}_{i}\right]} .
\end{aligned}
$$

Now we introduce the density field $\psi(\mathbf{x})$

$$
\psi(\mathbf{x})=\sum_{i} \delta\left(\mathbf{x}_{i}-\mathbf{x}\right)
$$

and the auxiliary field $\hat{\psi}(\mathbf{x})$. We can thus write the thermodynamics as

$$
Z_{\beta}=\int \mathcal{D} \psi(\mathbf{x}) \mathcal{D} \hat{\psi}(\mathbf{x}) e^{-G[\psi, \hat{\psi}]} .
$$

In terms of the field $\psi(\mathbf{x})$, the three terms $H_{0}, H_{1}$, and $H_{2}$ can be written as follows.

\section{1. $H_{0}$ term}

This is the usual two-body term that can be coarse grained as follows:

$$
H_{0}[\psi]=\frac{1}{2} \int d \mathbf{x} d \mathbf{y} \rho(\mathbf{x}) \phi(\mathbf{x}, \mathbf{y}) \rho(\mathbf{y}) .
$$




\section{2. $H_{1}$ term}

This term brings us to nonlinear and nonlocal interactions in the effective equilibrium theory. In particular, it is responsible for a $\psi^{3}$ term that explicitly breaks the symmetry $\psi \rightarrow-\psi$. This term can be rewritten as follows:

$$
\begin{aligned}
H_{1}[\psi] & =\frac{\tau}{8} \sum_{i}\left[\frac{\partial}{\partial \mathbf{x}_{i}} \sum_{k, l} \phi\left(\mathbf{x}_{k}, \mathbf{x}_{l}\right)\right]^{2} \\
& =\frac{\tau}{8} \sum_{i} 4\left[\sum_{k, l} \frac{\partial \phi\left(\mathbf{x}_{k}, \mathbf{x}_{l}\right)}{\partial \mathbf{x}_{k}} \frac{\partial \mathbf{x}_{k}}{\partial \mathbf{x}_{i}}\right]^{2} \\
& =\frac{\tau}{2} \sum_{i}\left[\sum_{k, l} \frac{\partial \phi\left(\mathbf{x}_{k}, \mathbf{x}_{l}\right)}{\partial \mathbf{x}_{k}} \delta_{k i}\right]^{2} \\
& \left.=\frac{\tau}{2} \sum_{i}\left[\sum_{l} \frac{\partial \phi\left(\mathbf{x}_{i}, \mathbf{x}_{l}\right)}{\partial \mathbf{x}_{i}}\right]^{2}\right]^{2} \\
& =\frac{\tau}{2} \sum_{i}\left[\int d \mathbf{x} \psi(\mathbf{x}) \partial_{\mathbf{x}_{i}} \phi\left(\mathbf{x}_{i}, \mathbf{x}\right)\right]^{\tau} \\
& =\frac{\tau}{2} \sum_{i}\left[\int d \mathbf{x} d \mathbf{y} \psi(\mathbf{x}) \psi(\mathbf{y}) \partial_{\mathbf{x}_{i}} \phi\left(\mathbf{x}_{i}, \mathbf{x}\right) \partial_{\mathbf{x}_{i}} \phi\left(\mathbf{x}_{i}, \mathbf{y}\right)\right] \\
& =\frac{\tau}{2} \int d \mathbf{x} d \mathbf{y} d \mathbf{z} \psi(\mathbf{x}) \psi(\mathbf{y}) \psi(\mathbf{z}) \partial_{\mathbf{z}} \phi(\mathbf{z}, \mathbf{x}) \partial_{\mathbf{z}} \phi(\mathbf{z}, \mathbf{y})
\end{aligned}
$$

where we have introduced the notation $\partial_{\mathbf{z}} \phi(\mathbf{z}, \mathbf{x}) \equiv \frac{\partial \phi(\mathbf{z}, \mathbf{x})}{\partial \mathbf{z}}$.

\section{3. $\mathrm{H}_{2}$ term}

In order to perform the coarse graining of the interactions due to $\mathrm{H}_{2}$, we have to deal with the determinant of a $d N \times$ $d N$ matrix. For doing that, let us assume a small $\tau$ limit; in this case, indicating with $\mathbf{H}$ the Hessian matrix and with $\mathbf{1}$ the identity matrix, one has

$$
\operatorname{det}(\mathbf{1}+\tau \mathbf{H}) \simeq 1+\tau \operatorname{Tr} \mathbf{H} .
$$

In this limit we can write

$$
H_{2}[\psi]=-\tau T \int d \mathbf{x} d \mathbf{y} \psi(\mathbf{x}) \partial_{\mathbf{x}, \mathbf{y}}^{2} \phi(\mathbf{x}, \mathbf{y}) \psi(\mathbf{y})
$$

where we have defined $\partial_{\mathbf{x}, \mathbf{y}}^{2} \phi(\mathbf{x}, \mathbf{y}) \equiv \frac{\partial^{2} \phi(\mathbf{x}, \mathbf{y})}{\partial \mathbf{x} \partial \mathbf{y}}$.

To prove that, we write the determinant of a generic matrix M of elements $M_{i j}$ as

$$
\operatorname{det} M_{i j}=\int \prod_{i} d \theta_{i} d \bar{\theta}_{i}, e^{\theta_{i} M_{i j} \bar{\theta}_{j}}
$$

where we have introduced two sets of conjugated Grassmann variables $\theta_{i}$ and $\bar{\theta}_{i}$ [40], respectively, that satisfy the anticommutation rules

$$
\begin{aligned}
& \left\{\theta_{i}, \theta_{j}\right\}=0, \\
& \left\{\bar{\theta}_{i}, \bar{\theta}_{j}\right\}=0 .
\end{aligned}
$$

Since $\theta_{i}$ and $\bar{\theta}_{i}$ are Grassmann variables, one has the following rules for the Grassmann integration:

$$
\begin{aligned}
\int d \theta_{i} d \bar{\theta}_{i} \bar{\theta}_{i} \theta_{i} & =1, \\
\int d \bar{\theta}_{i} \theta_{i} & =\int d \theta_{i} \bar{\theta}_{i}=0 .
\end{aligned}
$$

In our case $M_{i j}=\delta_{i j}+\tau H_{i j}$ and the determinant reads

$$
\begin{aligned}
\operatorname{det} M_{i j} & =\int \prod_{i} d \theta_{i} d \bar{\theta}_{i} e^{\theta_{i} \bar{\theta}_{i}+\tau \theta_{i} H_{i j} \bar{\theta}_{j}} \\
& =\int \prod_{i} d \theta_{i} d \bar{\theta}_{i} e^{\theta_{i} \bar{\theta}_{i}+\tau \theta_{i} \frac{\partial^{2} \phi}{\partial \mathbf{x}_{i} \partial \mathbf{x}_{j}} \bar{\theta}_{j}} \\
& =\int \prod_{i} d \theta_{i} d \bar{\theta}_{i} e^{\theta_{i} \bar{\theta}_{i}+\tau \int d \mathbf{x} d \mathbf{y} \theta_{i} \delta\left(\mathbf{x}-\mathbf{x}_{i}\right) \frac{\partial^{2} \phi}{\partial \mathbf{x} \partial \mathbf{y}} \delta\left(\mathbf{y}-\mathbf{y}_{j}\right) \bar{\theta}_{j}} .
\end{aligned}
$$

We now perform a small $\tau$ expansion that brings us to

$$
\begin{aligned}
\operatorname{det} M_{i j}= & \int \prod_{i} d \theta_{i} d \bar{\theta}_{i}\left[\theta_{i} \bar{\theta}_{i}\right. \\
& \left.+\tau \int d \mathbf{x} d \mathbf{y} \theta_{i} \delta\left(\mathbf{x}-\mathbf{x}_{i}\right) \frac{\partial^{2} \phi}{\partial \mathbf{x} \partial \mathbf{y}} \delta\left(\mathbf{y}-\mathbf{y}_{j}\right) \bar{\theta}_{j}\right]+O\left(\tau^{2}\right) \\
= & 1+\tau \int d \mathbf{x} d \mathbf{y} \psi(\mathbf{x}) \frac{\partial^{2} \phi(x, y)}{\partial \mathbf{x} \partial \mathbf{y}} \psi(\mathbf{y})+O\left(\tau^{2}\right) .
\end{aligned}
$$

Now we can write the following equilibrium action:

$$
\begin{aligned}
S_{\mathrm{eff}}[\psi]= & \frac{1}{2} \int d \mathbf{x} d \mathbf{y} \psi(\mathbf{x}) A(\mathbf{x}, \mathbf{y}) \psi(\mathbf{y}) \\
& +\frac{\tau}{2} \int d \mathbf{x} d \mathbf{y} d \mathbf{z} \psi(\mathbf{x}) \psi(\mathbf{y}) \psi(\mathbf{z}) B(\mathbf{x}, \mathbf{y}, \mathbf{z}) \\
A(x, y) \equiv & \phi(\mathbf{x}, \mathbf{y})-2 \tau T \partial_{\mathbf{x}, \mathbf{y}}^{2} \phi(\mathbf{x}, \mathbf{y}) \\
B(x, y, z) \equiv & \partial_{\mathbf{z}} \phi(\mathbf{z}, \mathbf{x}) \partial_{\mathbf{z}} \phi(\mathbf{z}, \mathbf{y})
\end{aligned}
$$

To obtain the thermodynamics of the model we have to compute the following partition function:

$$
Z=\int \mathcal{D} \psi(\mathbf{x}) \mathcal{D} \hat{\psi}(\mathbf{x}) e^{-\int d \mathbf{x} \psi(\mathbf{x}) \hat{\psi}(\mathbf{x})+N \ln z-S_{\text {eff }}[\psi]}
$$

where we have introduced the "single-particle" partition function $z \equiv \int d \mathbf{x} e^{\hat{\psi}(\mathbf{x})}$. Let us rewrite the action as follows:

$$
G[\psi, \hat{\psi}] \equiv \int d \mathbf{x} \psi(\mathbf{x}) \hat{\psi}(\mathbf{x})-N \ln z+S_{\mathrm{eff}}[\psi]
$$

and thus

$$
Z=\int \mathcal{D} \psi(\mathbf{x}) \mathcal{D} \hat{\psi}(\mathbf{x}) e^{-G[\psi, \hat{\psi}]} .
$$

The functional integral can be evaluated using the saddlepoint approximation that allows us to write

$$
Z \sim e^{-G[\rho, \hat{\rho}]}
$$


where the fields $\rho(\mathbf{x})$ and $\hat{\rho}(\mathbf{x})$ satisfy the saddle-point equations

$$
\begin{aligned}
& \left.\frac{\delta G}{\delta \psi}\right|_{\rho, \hat{\rho}}=\hat{\rho}+\frac{\delta S_{\text {eff }}}{\delta \rho}=0, \\
& \left.\frac{\delta G}{\delta \hat{\psi}}\right|_{\rho, \hat{\rho}}=\rho-\frac{N e^{\hat{\rho}}}{\int d \mathbf{x} e^{\hat{\rho}}}=0 .
\end{aligned}
$$

Using the first equation for eliminating $\hat{\rho}$, the free energy of the model reads

$$
\begin{aligned}
G[\rho]= & S_{\text {eff }}[\rho]+\int d \mathbf{x} \rho(\mathbf{x}) \ln \rho(\mathbf{x})-N \ln N, \\
S_{\mathrm{eff}}[\rho]= & \frac{1}{2} \int d \mathbf{x} d \mathbf{y} \rho(\mathbf{x}) A(\mathbf{x}, \mathbf{y}) \rho(\mathbf{y}) \\
& +\frac{\tau}{2} \int d \mathbf{x} d \mathbf{y} d \mathbf{z} \rho(\mathbf{x}) \rho(\mathbf{y}) \rho(\mathbf{z}) B(\mathbf{x}, \mathbf{y}, \mathbf{z}) .
\end{aligned}
$$

The action (E20) reduces to the equilibrium case for $\tau \rightarrow 0$. Moreover, for $\tau \neq 0$ the theory includes a $\rho^{3}$ term.

\section{APPENDIX F: COMPUTATION OF THE EFFECTIVE ACTION IN THE CASE OF CENTRAL PAIR POTENTIALS}

In the case of central pair potentials, one has

$$
\phi\left(\mathbf{x}_{i}, \mathbf{x}_{j}\right)=\phi\left(\left|\mathbf{x}_{i}-\mathbf{x}_{j}\right|\right)=\phi\left(r_{i j}\right)
$$

where we have defined $r_{i j} \equiv\left|\mathbf{x}_{i}-\mathbf{x}_{j}\right|$. In $d$ spatial dimensions, the vector $\mathbf{x}_{i}$ is individuated by its components $\mathbf{x}_{i}=$ $\left\{x_{i}^{\alpha}\right\}_{\alpha=1}^{d}$ and the Euclidean distance reads

$$
r_{i j}^{2}=\sum_{\alpha=1}^{d}\left(x_{i}^{\alpha}-x_{j}^{\alpha}\right)
$$

The first derivative of $\phi\left(r_{i j}\right)$ with respect to $\mathbf{x}_{i}$ is

$$
\frac{\partial \phi\left(\mathbf{x}_{i}, \mathbf{x}_{j}\right)}{\partial \mathbf{x}_{i}}=\frac{1}{r_{i j}} \frac{\partial \phi}{\partial r_{i j}}=\frac{\phi^{\prime}\left(r_{i j}\right)}{r_{i j}}
$$

where the prime indicates the derivative with respect to $r$ in $\phi(r)$. The Hessian matrix $\mathbf{H}$ of components $H_{i j}^{\alpha \beta}$ is

$$
H_{i j}^{\alpha \beta}=\sum_{l}\left[\frac{\partial^{2} \phi}{\partial r_{l i}} \frac{\partial r_{l i}}{\partial x_{j}^{\beta}} \frac{\partial r_{l i}}{\partial x_{i}^{\alpha}}+\frac{\partial \phi}{\partial r_{l i}} \frac{\partial^{2} r_{l i}}{\partial x_{i}^{\alpha} \partial x_{j}^{\beta}}\right]
$$

and the diagonal part is

$$
\begin{aligned}
H_{i i}^{\alpha \alpha}= & \sum_{l}\left\{\frac{\phi^{\prime \prime}\left(r_{l i}\right)}{r_{l i}^{2}}\left(x_{l}^{\alpha}-x_{i}^{\alpha}\right)^{2}\right. \\
& \left.+\phi^{\prime}\left(r_{l i}\right)\left[\frac{1}{r_{l i}}-\frac{\left(x_{l}^{\alpha}-x_{i}^{\alpha}\right)^{2}}{r_{l i}^{3}}\right]\right\} .
\end{aligned}
$$

The trace of $\mathbf{H}$ is

$$
\operatorname{Tr} \mathbf{H}=\sum_{i, \alpha} H_{i i}^{\alpha \alpha}=\sum_{l, i}\left[\phi^{\prime \prime}\left(r_{l i}\right)+\frac{\phi^{\prime}\left(r_{l i}\right)}{r_{l i}}(d-1)\right] .
$$

In terms of the local-density field $\psi(\mathbf{x})$ we can thus write

$$
\begin{aligned}
H_{2}[\psi] & =-\tau T \int d \mathbf{x} d \mathbf{y} \psi(\mathbf{x}) f(|\mathbf{x}-\mathbf{y}|) \psi(\mathbf{y}), \\
f(r) & \equiv \phi^{\prime \prime}(r)+\frac{\phi^{\prime}(r)}{r}(d-1) .
\end{aligned}
$$

The term $H_{1}\left[\mathbf{x}_{i}\right]$ is

$$
\begin{aligned}
H_{1}[\psi]= & \frac{\tau}{2} \sum_{i}\left(\nabla_{\mathbf{x}_{i}} H_{0}\right) \cdot\left(\nabla_{\mathbf{x}_{i}} H_{0}\right) \\
= & \frac{\tau}{2} \sum_{i}\left(\sum_{l} \frac{1}{r_{i l}} \frac{\partial \phi}{\partial r_{i l}}\left(\mathbf{x}_{i}-\mathbf{x}_{l}\right)\right) \\
& \cdot\left(\sum_{m} \frac{1}{r_{i m}} \frac{\partial \phi}{\partial r_{i m}}\left(\mathbf{x}_{i}-\mathbf{x}_{m}\right)\right) \\
= & \frac{\tau}{2} \sum_{i, l, m} \frac{\left(\mathbf{x}_{i}-\mathbf{x}_{l}\right) \cdot\left(\mathbf{x}_{i}-\mathbf{x}_{m}\right)}{r_{i l} r_{i m}} \frac{\partial \phi}{\partial r_{i l}} \frac{\partial \phi}{\partial r_{i m}} \\
= & \frac{\tau}{2} \int d \mathbf{x} d \mathbf{y} d \mathbf{z} \frac{(\mathbf{x}-\mathbf{y}) \cdot(\mathbf{x}-\mathbf{z})}{r_{x y} r_{x z}} \\
& \times \phi^{\prime}\left(r_{x y}\right) \phi^{\prime}\left(r_{x z}\right) \psi(\mathbf{x}) \psi(\mathbf{y}) \psi(\mathbf{z}) .
\end{aligned}
$$

At the saddle point we have $\langle\psi(\mathbf{x})\rangle=\rho(\mathbf{x})$ and thus we can finally write the action for central potentials:

$$
\begin{aligned}
G[\rho]= & S_{\mathrm{eff}}[\rho]+\int d \mathbf{x} \rho(\mathbf{x}) \ln \rho(\mathbf{x}), \\
S_{\mathrm{eff}}[\rho] \equiv & \int d \mathbf{x} d \mathbf{y} \rho(\mathbf{x}) A(|\mathbf{x}-\mathbf{y}|) \rho(\mathbf{y}) \\
& +\frac{\tau}{2} \int d \mathbf{x} d \mathbf{y} d \mathbf{z} B(|\mathbf{x}-\mathbf{y}|,|\mathbf{x}-\mathbf{z}|) \rho(\mathbf{x}) \rho(\mathbf{y}) \rho(\mathbf{z})
\end{aligned}
$$

where the function $A(r)$ now reads

$$
A(r)=\phi(r)-\tau T f(r)
$$

and the function $B(r, s)$ is

$$
B(r, s)=\frac{\mathbf{r} \cdot \mathbf{s}}{r s} \phi^{\prime}(r) \phi^{\prime}(s) .
$$

\section{APPENDIX G: LATTICE MODELS AND THE CONTINUUM LIMIT FOR $H_{\mathrm{UCN}}$}

Here we perform the coarse-graining scheme introduced in Sec. VIII in the case of $H_{\mathrm{UCN}}$. Again, we consider the system composed by $N$ particles, each one of volume $\delta$. Particles are confined in a box of volume $V$. We divide the volume in a small region of volume $\Delta$, each region containing $N_{i}$ particles 
with $\sum_{i} N_{i}=N$. The partition function reads

$$
\begin{aligned}
Z_{\beta}= & \sum_{\left\{N_{i}\right\} \mid \sum_{i} N_{i}=N} e^{-G\left[N_{i}\right]}, \\
-G\left[N_{i}\right] \equiv & \sum_{i, j} \mathcal{A}_{i j} N_{i} N_{j}+\frac{\tau}{2} \sum_{i, l, m} \mathcal{B}_{i l m} N_{i} N_{l} N_{m} \\
& +\sum_{i}\left[N_{i} \ln \left(\Delta-N_{i} \delta\right)-N_{i} \ln N_{i}+N_{i}\right], \\
\mathcal{A}_{i j} \equiv & \beta \phi_{i j}-\tau f_{i j}, \\
\mathcal{B}_{i l m} \equiv & \phi_{i l}^{\prime} \phi_{i m}^{\prime} \frac{\left(\mathbf{r}_{i}-\mathbf{r}_{l}\right)}{r_{i l}} \cdot \frac{\left(\mathbf{r}_{i}-\mathbf{r}_{m}\right)}{r_{i m}} .
\end{aligned}
$$

The continuum limit is obtained considering $\Delta \rightarrow 0$ that brings us to

$$
\begin{aligned}
-G[\rho]= & \int d \mathbf{x} d \mathbf{y} \mathcal{A}(|\mathbf{x}-\mathbf{y}|) \rho(\mathbf{x}) \rho(\mathbf{y}) \\
& +\frac{\tau}{2} \int d \mathbf{x} d \mathbf{y} d \mathbf{z} \mathcal{B}(|\mathbf{x}-\mathbf{y}|,|\mathbf{x}-\mathbf{z}|) \rho(\mathbf{x}) \rho(\mathbf{y}) \rho(\mathbf{z}) \\
& +\int d \mathbf{x}\left[1+\ln \left(\frac{1-\rho(\mathbf{x})}{\rho(\mathbf{x})}\right)\right]
\end{aligned}
$$

\section{Mean-field theories with a cubic term}

We consider homogeneous solutions described by a real number $\rho \in[0,1]$. Now the action reads

$$
-g(\rho)=\alpha \rho^{2}+\frac{\tau b}{2} \rho^{3}+\rho\left[\ln \frac{1-\rho}{\rho}+1\right] .
$$

To make our discussion the simplest possible without loss of generality, we consider only two control parameters that are $\alpha$ and $\tau b / 2 \rightarrow b$. In particular, we are interested in evaluating the impact that the cubic term has on spinodal decomposition. For this reason we will consider the situation $\alpha<0$. The coexistence region in the $T$ vs $\rho$ plane, obtained considering the solution of $\partial_{\rho} P=0$, is

$$
T(\rho, b)=2 \rho(1-\rho)^{2}(3 b \rho-\alpha),
$$

that has been obtained considering $\alpha=-1$. The curves $T(\rho, b)$ are shown in Fig. 3 (left panel) for different values of the asymmetry parameter $b$. As $b$ increases we obtain a contraction of the coexistence region. We can now compute the location of the critical point that is obtained considering the set of equations $\partial_{\rho} P=\partial_{\rho}^{2} P=0$. Once we get the critical density $\rho_{c}(b)$, that is shown in Fig. 3, central panel, we can also compute how the critical temperature changes with $b$ (same figure, right panel). It turns that both the critical density and the critical temperature are decreasing functions of $b$.

\section{Landau-Ginzburg $\varphi^{3}$ theory}

We provide here a brief discussion about $\varphi^{3}$ field theories in statistical physics. In particular, we consider both interactions $\varphi^{3}$ and $\varphi^{4}$ in order to have a well-defined LandauGinzburg energy functional. For $b>0$, the critical behavior of the system can be studied through the following Landau-
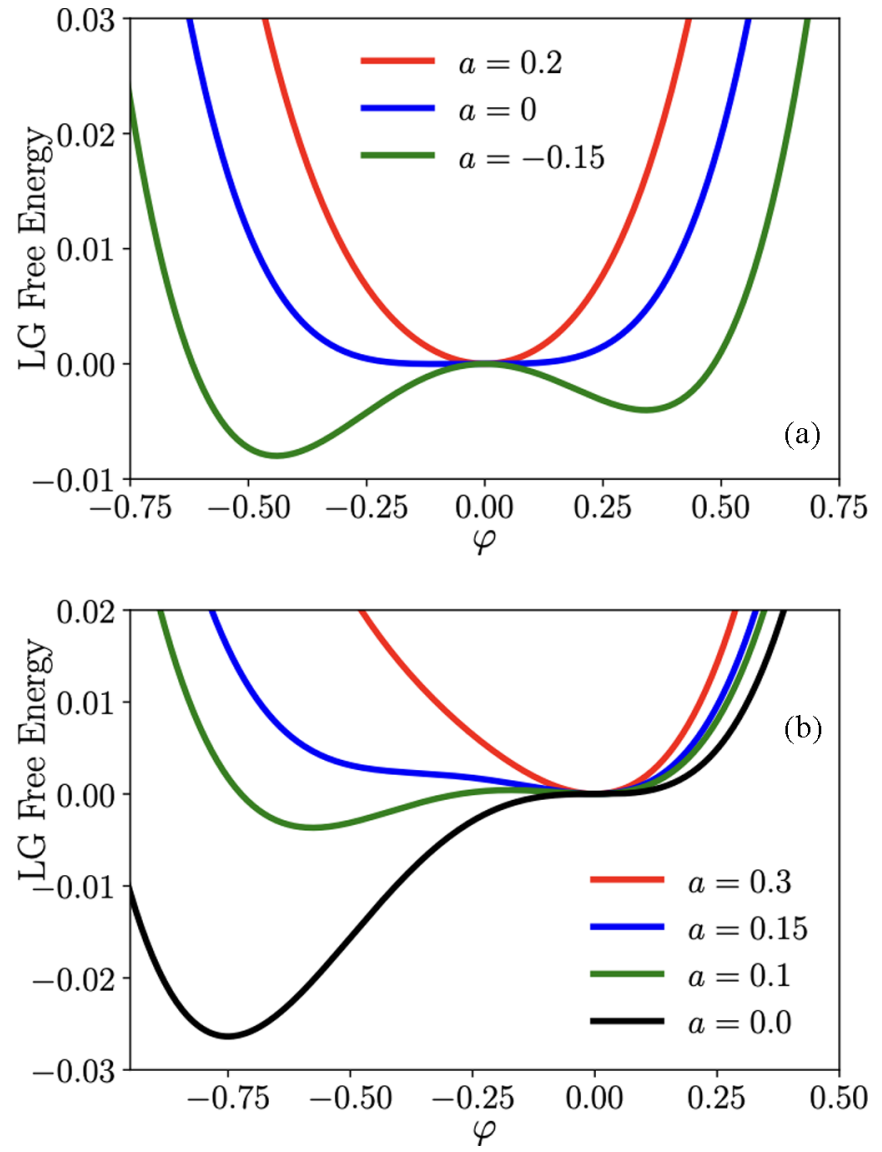

FIG. 7. Landau-Ginzburg free energy (G6) for $b^{2}<4 a$ at (a) $a>0$ and (b) $b^{2} \geqslant 4 a$.

Ginzburg free energy:

$$
F_{\mathrm{LG}}[\varphi]=\int d \mathbf{x}\left[\frac{1}{2}(\nabla \varphi)^{2}+\frac{a}{2} \varphi(\mathbf{x})^{2}+\frac{b}{3} \varphi(\mathbf{x})^{3}+\frac{c}{4} \varphi(\mathbf{x})^{4}\right]
$$

where the order parameter $\varphi(\mathbf{x})$ represents fluctuations around the critical density $\rho_{c}$, i.e., $\rho(\mathbf{x})=\rho_{c}+\varphi(\mathbf{x})$. Without loss of generality, we consider the homogeneous case $\varphi(\mathbf{x})=\varphi$. We immediately realize that the cubic term, breaking the symmetry $\varphi \rightarrow-\varphi$, promotes one phase with respect to the other [57]. Minimizing Eq. (G5), one obtains three configurations $\varphi_{0,1,2}$ :

$$
\begin{aligned}
\varphi_{0} & =0, \\
\varphi_{1,2} & =\frac{-b}{2 c} \pm \frac{1}{2 c} \sqrt{b^{2}-4 a c} .
\end{aligned}
$$

For the sake of simplicity, let us fix $c=1$. We consider the coefficients $a$ and $b$ as two independent and tunable external parameters of our coarse-grained model. For $b=0$, the coefficient $a$ changes sign at the MIPS critical point. For $b \neq 0$ the value $a=0$ is not necessarily the MIPS critical point. In particular, the coefficient $b$ tunes the intensity of asymmetry $\varphi \rightarrow-\varphi$. For $b=0$, we recover the standard $\varphi^{4}$ theory, and thus the symmetry $\varphi \rightarrow-\varphi$ is preserved. For 
$b>0$ and $b^{2}<4 a$, at $a>0$ the only real and stable solution is $\varphi_{0}$ that becomes marginal at $a=0$ and eventually unstable for $a<0$. In the latter case, $\varphi_{1,2}$ becomes real and the solution $\varphi_{1}$ is the new minimum. When $b^{2} \geqslant 4 a, \varphi_{1,2}$ are real for $a>0$, meaning that the system develops a metastable state. The two situations are shown in Fig. 7.

\section{APPENDIX H: NUMERICAL SIMULATIONS}

We performed numerical simulations of an active gas in one dimension embedded into a confining external field $A(x)=x^{4} / 4$. The equation of motion for the particle $i$, with

$$
\begin{aligned}
& i=1, \ldots, N \text {, is } \\
& \dot{x}_{i}=-\mu \frac{d A}{d x_{i}}+\mu f_{i}, \quad \tau \dot{f_{i}}=-f_{i}+\eta_{i}
\end{aligned}
$$

with $\left\langle\eta_{i}\right\rangle=0$ and $\left\langle\eta_{i}(t) \eta_{j}(s)\right\rangle=2 T \delta_{i j} \delta(t-s)$. $f_{i}$ is the active force; $\tau$ is the correlation time of the noise. We solved Eq. (H1) numerically using the Euler method. We have considered a gas of $N=4000$ particles with $T=1$, $\mu=1$, and $\tau=1$. The density distribution in the steady state $P_{\text {num }}(x)$ has been computed through the histogram of $x_{i}\left(t^{*}\right)$, where $t^{*}$ is chosen large enough for guaranteeing $t^{*} \gg \tau$.
[1] A. Klopper, Nat. Phys. 14, 645 (2018).

[2] X. Trepat and E. Sahai, Nat. Phys. 14, 671 (2018).

[3] H.-P. Zhang, A. Be'er, E.-L. Florin, and H. L. Swinney, Proc. Natl. Acad. Sci. USA 107, 13626 (2010).

[4] O. Feinerman, I. Pinkoviezky, A. Gelblum, E. Fonio, and N. S. Gov, Nat. Phys. 14, 683 (2018).

[5] C. Bechinger, R. Di Leonardo, H. Löwen, C. Reichhardt, G. Volpe, and G. Volpe, Rev. Mod. Phys. 88, 045006 (2016).

[6] M. C. Marchetti, J. F. Joanny, S. Ramaswamy, T. B. Liverpool, J. Prost, M. Rao, and R. A. Simha, Rev. Mod. Phys. 85, 1143 (2013).

[7] S. Ramaswamy, Annu. Rev. Condens. Matter Phys. 1, 323 (2010).

[8] M. E. Cates, Rep. Prog. Phys. 75, 042601 (2012).

[9] A. Cavagna and I. Giardina, Annu. Rev. Condens. Matter Phys. 5, 183 (2014).

[10] M. Ballerini, N. Cabibbo, R. Candelier, A. Cavagna, E. Cisbani, I. Giardina, V. Lecomte, A. Orlandi, G. Parisi, A. Procaccini et al., Proc. Natl. Acad. Sci. USA 105, 1232 (2008).

[11] C. Malinverno, S. Corallino, F. Giavazzi, M. Bergert, Q. Li, M. Leoni, A. Disanza, E. Frittoli, A. Oldani, E. Martini et al., Nat. Mater. 16, 587 (2017).

[12] J. Bialké, T. Speck, and H. Löwen, J. Non-Cryst. Solids 407, 367 (2015)

[13] J. Bialké, H. Löwen, and T. Speck, Europhys. Lett. 103, 30008 (2013).

[14] J. Bialké, T. Speck, and H. Löwen, Phys. Rev. Lett. 108, 168301 (2012).

[15] J. Stenhammar, D. Marenduzzo, R. J. Allen, and M. E. Cates, Soft Matter 10, 1489 (2014).

[16] G. Szamel, E. Flenner, and L. Berthier, Phys. Rev. E 91, 062304 (2015).

[17] Y. Fily and M. C. Marchetti, Phys. Rev. Lett. 108, 235702 (2012).

[18] L. Berthier and J. Kurchan, Nat. Phys. 9, 310 (2013).

[19] T. Arnoulx de Pirey, G. Lozano, and F. van Wijland, Phys. Rev. Lett. 123, 260602 (2019).

[20] T. B. Liverpool and M. C. Marchetti, Phys. Rev. Lett. 90, 138102 (2003).

[21] F. C. Keber, E. Loiseau, T. Sanchez, S. J. DeCamp, L. Giomi, M. J. Bowick, M. C. Marchetti, Z. Dogic, and A. R. Bausch, Science 345, 1135 (2014).
[22] C. Maggi, U. M. B. Marconi, N. Gnan, and R. Di Leonardo, Sci. Rep. 5, 10742 (2015).

[23] G. Szamel, Phys. Rev. E 90, 012111 (2014).

[24] N. Koumakis, C. Maggi, and R. Di Leonardo, Soft matter 10, 5695 (2014).

[25] P. Jung and P. Hänggi, Phys. Rev. A 35, 4464 (1987).

[26] P. Hänggi and P. Jung, Adv. Chem. Phys. 89, 239 (1995).

[27] J. Rammer, Quantum Field Theory of Non-Equilibrium States (Cambridge University Press, 2007)

[28] R. Wittkowski, A. Tiribocchi, J. Stenhammar, R. J. Allen, D. Marenduzzo, and M. E. Cates, Nat. Commun. 5, 4351 (2014).

[29] C. Nardini, É. Fodor, E. Tjhung, F. van Wijland, J. Tailleur, and M. E. Cates, Phys. Rev. X 7, 021007 (2017).

[30] F. Caballero, C. Nardini, and M. E. Cates, J. Stat. Mech.: Theory Exp. (2018) 123208.

[31] A. Tiribocchi, R. Wittkowski, D. Marenduzzo, and M. E. Cates, Phys. Rev. Lett. 115, 188302 (2015).

[32] J. Stenhammar, A. Tiribocchi, R. J. Allen, D. Marenduzzo, and M. E. Cates, Phys. Rev. Lett. 111, 145702 (2013).

[33] T. Speck, J. Bialké, A. M. Menzel, and H. Löwen, Phys. Rev. Lett. 112, 218304 (2014).

[34] A. M. Menzel, A. Saha, C. Hoell, and H. Löwen, J. Chem. Phys. 144, 024115 (2016).

[35] J. M. Kosterlitz and D. J. Thouless, J. Phys. C 6, 1181 (1973).

[36] B. I. Halperin and D. R. Nelson, Phys. Rev. Lett. 41, 121 (1978).

[37] A. P. Young, Phys. Rev. B 19, 1855 (1979).

[38] J. Tailleur and M. E. Cates, Phys. Rev. Lett. 100, 218103 (2008).

[39] M. Plischke and B. Bergersen, Equilibrium Statistical Physics (World Scientific, Singapore, 1994).

[40] J. Zinn-Justin, Quantum Field Theory and Critical Phenomena (Clarendon, Oxford, 1996).

[41] G. Parisi, Statistical Field Theory (Addison-Wesley, Reading, MA, 1988)

[42] T. F. F. Farage, P. Krinninger, and J. M. Brader, Phys. Rev. E 91, 042310 (2015).

[43] U. M. B. Marconi and C. Maggi, Soft Matter 11, 8768 (2015).

[44] U. M. B. Marconi, N. Gnan, M. Paoluzzi, C. Maggi, and R. Di Leonardo, Sci. Rep. 6, 23297 (2016).

[45] U. M. B. Marconi, M. Paoluzzi, and C. Maggi, Mol. Phys. 114, 2400 (2016).

[46] G. Szamel, Phys. Rev. E 93, 012603 (2016).

[47] U. M. B. Marconi, C. Maggi, and M. Paoluzzi, arXiv: 1705.02481 . 
[48] M. Paoluzzi, U. M. B. Marconi, and C. Maggi, Phys. Rev. E 97, 022605 (2018).

[49] C. Maggi, M. Paoluzzi, L. Angelani, and R. Di Leonardo, Sci. Rep. 7, 17588 (2017).

[50] D. T. N. Chen, A. W. C. Lau, L. A. Hough, M. F. Islam, M. Goulian, T. C. Lubensky, and A. G. Yodh, Phys. Rev. Lett. 99, 148302 (2007).

[51] C. Maggi, M. Paoluzzi, N. Pellicciotta, A. Lepore, L. Angelani, and R. Di Leonardo, Phys. Rev. Lett. 113, 238303 (2014).

[52] S. Das, G. Gompper, and R. G. Winkler, New J. Phys. 20, 015001 (2018).

[53] L. Caprini, U. M. B. Marconi, and A. Puglisi, Sci. Rep. 9, 1386 (2019).

[54] F. J. Sevilla, A. V. Arzola, and E. P. Cital, Phys. Rev. E 99, 012145 (2019).

[55] I. D. Vladescu, E. J. Marsden, J. Schwarz-Linek, V. A. Martinez, J. Arlt, A. N. Morozov, D. Marenduzzo, M. E. Cates, and W. C. K. Poon, Phys. Rev. Lett. 113, 268101 (2014).

[56] N. G. van Kampen, Phys. Rev. 135, A362 (1964).
[57] J.-L. Barrat and J.-P. Hansen, Basic Concepts for Simple and Complex Liquids (Cambridge University, Cambridge, England, 2003).

[58] M. E. Cates and J. Tailleur, Annu. Rev. Condens. Matter Phys. 6, 219 (2015).

[59] M. Cates and J. Tailleur, Europhys. Lett. 101, 20010 (2013).

[60] A. P. Solon, J. Stenhammar, R. Wittkowski, M. Kardar, Y. Kafri, M. E. Cates, and J. Tailleur, Phys. Rev. Lett. 114, 198301 (2015).

[61] N. Schupper and N. M. Shnerb, Phys. Rev. Lett. 93, 037202 (2004).

[62] A. Crisanti and L. Leuzzi, Phys. Rev. Lett. 95, 087201 (2005).

[63] A. P. Hughes, U. Thiele, and A. J. Archer, Am. J. Phys. 82, 1119 (2014).

[64] G. Liu, A. Patch, F. Bahar, D. Yllanes, R. D. Welch, M. C. Marchetti, S. Thutupalli, and J. W. Shaevitz, Phys. Rev. Lett. 122, 248102 (2019).

[65] S. Hermann, P. Krinninger, D. de las Heras, and M. Schmidt, Phys. Rev. E 100, 052604 (2019). 